\title{
Effects of Time-Dependent Stimuli in a Competitive Neural Network Model of Perceptual Rivalry
}

\author{
Suren Jayasuriya $\cdot$ Zachary P. Kilpatrick
}

Received: 1 October 2011 / Accepted: 16 January 2012

(C) Society for Mathematical Biology 2012

\begin{abstract}
We analyze a competitive neural network model of perceptual rivalry that receives time-varying inputs. Time-dependence of inputs can be discrete or smooth. Spike frequency adaptation provides negative feedback that generates network oscillations when inputs are constant in time. Oscillations that resemble perceptual rivalry involve only one population being "ON" at a time, which represents the dominance of a single percept at a time. As shown in Laing and Chow (J. Comput. Neurosci. 12(1):39-53, 2002), for sufficiently high contrast, one can derive relationships between dominance times and contrast that agree with Levelt's propositions (Levelt in On binocular rivalry, 1965). Time-dependent stimuli give rise to novel network oscillations where both, one, or neither populations are "ON" at any given time. When a single population receives an interrupted stimulus, the fundamental mode of behavior we find is phase-locking, where the temporally driven population locks its state to the stimulus. Other behaviors are analyzed as bifurcations from this forced oscillation, using fast/slow analysis that exploits the slow timescale of adaptation. When both populations receive time-varying input, we find mixtures of fusion and sole population dominance, and we partition parameter space into particular oscillation types. Finally, when a single population's input contrast is smoothly varied in time, $1: n$ mode-locked states arise through period-adding bifurcations beyond phase-locking. Our results provide several testable predictions for future psychophysical experiments on perceptual rivalry.
\end{abstract}

Keywords Perceptual rivalry · Intermittent ambiguous stimuli · Mutual inhibition · Periodically forced $\cdot$ Mode-locking

\footnotetext{
S. Jayasuriya $\cdot$ Z.P. Kilpatrick $(\bowtie)$

Department of Mathematics, University of Pittsburgh, Pittsburgh, PA 15260, USA

e-mail: zpkilpat@pitt.edu

S. Jayasuriya

e-mail: smj20@pitt.edu
} 


\section{Introduction}

Perceptual rivalry is the visual phenomenon that occurs when the brain alternates between multiple interpretations of a visual stimulus. Binocular rivalry is the specific case where perception alternates back and forth between images presented to both eyes (see Fig. 1a-b). Usually, images presented to induce rivalry maintain the same contrast throughout the duration of a single experiment (Levelt 1965; Blake and Logothetis 2002). In this context, the durations of each percept or dominance times last a few seconds each. However, there are studies that account for effects of periodically interrupted stimuli (Orbach et al. 1963; Leopold et al. 2002; Chen and He 2004; Noest et al. 2007; Pearson and Brascamp 2008) and stimuli with time-dependent contrast (Mueller and Blake 1989; Wilson et al. 2001; Lankheet 2006; Kang et al. 2009). Dominance times can in fact increase from several seconds to several minutes if stimuli are interrupted rapidly enough (Leopold et al. 2002; Brascamp et al. 2008). Psychophysical observations of experiments with timedependent stimuli significantly extend insight provided by investigations with static images.

Relationships between stimulus contrast and dominance times in perceptual rivalry help determine the phenomenon's neural substrates. A seminal study of binocular rivalry invoked by static stimuli led to four propositions developed by Levelt (1965). Each proposition concerns an effect of altering the contrast of stimuli in subsequent experiments, not during the course of a single experiment: (i) increasing the contrast of one stimulus increases the proportion of time that stimulus is dominant; (ii) increasing the contrast of one stimulus does not affect its average dominance

(a) Necker cube

(b) Binocular gratings

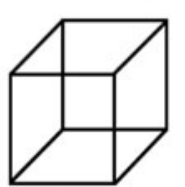

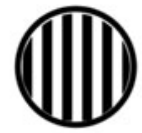

$\mathrm{L}$

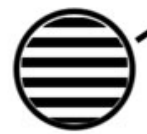

$\mathrm{R}$ decrease $\mathrm{L}$ contrast (c) Both inputs varied
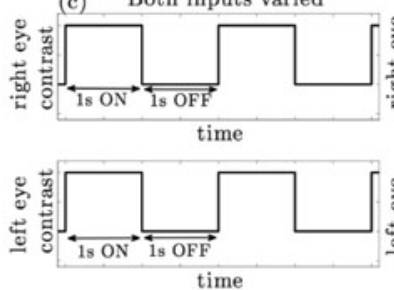

(d) Single input varied
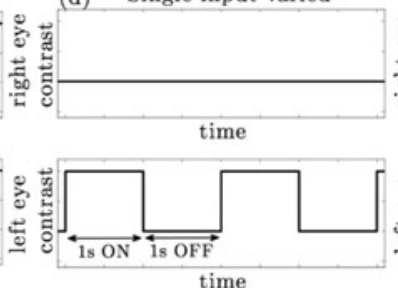

(e) Single input varied smoothly
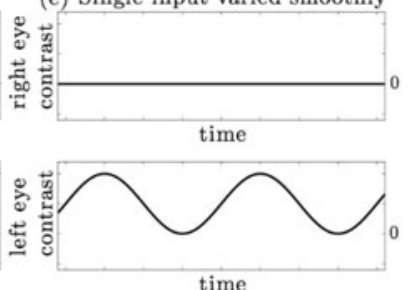

Fig. 1 Ambiguous visual inputs that generate perceptual rivalry. (a) Necker cube can be perceived as having either square face in front. (b) Presenting orthogonal gratings to either eye generates binocular rivalry. Decreasing static contrast of the left eye's stimulus increases the dominance time of the right eye percept and does not change that of the left eye percept, according to Levelt's propositions (Levelt 1965). (c) Interrupting both percepts in time can increase both percepts' dominance times (Orbach et al. 1963; Leopold et al. 2002; Noest et al. 2007). Specific cases of temporally varying a single percept's contrast by either (d) interrupting it or (e) smoothly changing it were studied in Mueller and Blake (1989), Laing and Chow (2002) 
time; (iii) increasing contrast of one stimulus increases the rivalry alternation rate; and (iv) increasing the contrast of both stimuli increases the rivalry alternation rate. Propositions (i)-(iii) imply that increasing the contrast of one eye's stimulus will decrease the dominance time of the other. Recent evidence suggests proposition (ii) may only hold for high contrast stimuli (Bossink et al. 1993), and increasing one stimulus' contrast affects the dominance time of the stronger stimulus (Brascamp et al. 2006; Klink et al. 2008; Moreno-Bote et al. 2010). There are also recent observations regarding dominance time dependence upon intermittently presented stimuli (see Fig. 1c). Periodically interrupted ambiguous images can be perceived in the same configuration several presentations in a row (Orbach et al. 1963; Leopold et al. 2002; Blake et al. 2003; Pearson and Brascamp 2008), possibly due to recovery of adaptive processes in the interruption period (Brascamp et al. 2009). Switching seems to not be noise-dominated, since rivalrous processes appear to have memory up to several minutes (Leopold et al. 2002; Pearson and Brascamp 2008). In addition, the periodic strengthening of contrast in the images of alternating eyes can provide a means by which perception can actually lock to the time course of these stimulus intensifications (Kang et al. 2009). Understanding the neural processes underlying these kinds of effects could have implications for many aspects of visual processing.

There are other complex dynamics that arise in perceptual rivalry experiments that have yet to be fully mined for information about the visual system. It has been shown that certain binocular stimuli can evoke four, rather than two, different perceptual representations that include fusions of either eye's image (Suzuki and Grabowecky 2002). Only recently have perceptual rivalry experiments used novel stimulus paradigms as a means of uncovering further information about the adaptive processes of the visual system (Brascamp et al. 2009; Webster 2011). Studying complex perceptual dynamics in psychophysical experiments may provide clues as to the relationship between visual channels for binocular rivalry and stereopsis (Wolfe 1986). In Buckthought et al. (2008), an examination of hysteresis between rivalrous behavior and fusion of binocular images quantified the critical stimulus disparity necessary to generate rivalry. Such studies work toward maximizing the capability of noninvasive protocols for quantifying stereoscopic visual processing.

Mathematical models that describe perceptual rivalry commonly use multistable neural competition where two populations have self-excitatory and mutually inhibitory connections (Wang and Rinzel 1992; Seely and Chow 2011). Switches in population dominance occur through slow negative feedback processes commonly implemented as synaptic depression and/or spike frequency adaptation. Synaptic depression is the process by which synaptic resources such as neurotransmitters and vesicles are exhausted due to continuous use (Abbott et al. 1997). Spike frequency adaptation arises through a hyperpolarizing current that decreases a neuron's instantaneous firing rate (Benda and Herz 2003). Feedback causes switches through either a release or escape mechanism (Wang and Rinzel 1992; Curtu et al. 2008). In release, the dominant population's inhibition of the suppressed becomes weak through its own negative feedback. In escape, the suppressed population overcomes the dominant's inhibition through its own recovery. Mutual inhibition mod- 


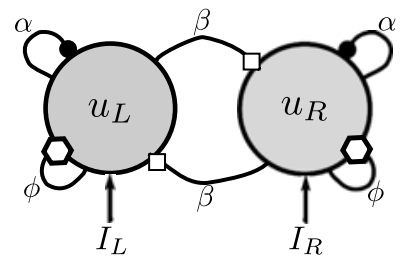

Fig. 2 Competitive neural network receiving time-dependent inputs $I_{L}$ and $I_{R}$, described in the system (1a)-(1d). Our paradigm allows for both or either input to the neural populations $u_{L}$ and $u_{R}$ to vary in time. Self-excitation (black dots) has strength $\alpha$; cross-inhibition (white squares) has strength $\beta$; and self-adaptation (white hexagons) has strength $\phi$

els have grown to be considered a sufficient description of the basic mechanisms involved in perceptual rivalry as they are successful in reproducing Levelt's propositions (Laing and Chow 2002; Taylor et al. 2002; Wilson 2003; Shpiro et al. 2007; Curtu et al. 2008; Seely and Chow 2011). Existing theoretical models of perceptual rivalry have been modified in ways to account for how interrupted stimuli can lengthen effective dominance times (Leopold et al. 2002; Noest et al. 2007; Brascamp et al. 2009). A common feature of these models is some slow adaptive process that contains the memory of the previous percept orientation during periods of stimulus interruption. In Gigante et al. (2009), a multistage stochastic model was used to account for multiple peaks in the frequency distribution of dominance times for stimuli with periodic ON and OFF epochs. These studies mostly examine the case where the timescale of interruption is significantly shorter than the timescale of percept switch. However, cases where the timescales of interruption and dominance switching start to interact may be of interest as a further touchstone for the validity of models of perceptual rivalry.

Thus, we seek to extend the present results on theoretical models of perceptual rivalry by examining the effects of various time-dependent stimuli on the behavior of a mutual inhibitory network with slow adaptation (Fig. 2 and Sect. 2). We first analyze several behaviors that arise in the model derived by Laing and Chow (2002) with constant stimuli and show it can support various complex behaviors without timedependence in its inputs (Sect. 3). Then we examine effects of periodically varying a single percept's contrast (Sect. 4) and give conditions for a simple mode of behavior where the population is dominant when its time-varying input is ON and suppressed when its input is OFF. In addition, we explore a variety of more complex periodic solutions that result as the timescale and amplitude of the stimulus is modified. Upon varying both populations' inputs simultaneously, we find many more new complex modes of behavior (Sect. 5). Our analysis allows us to construct numerically verified diagrams that partition parameter space into these different complex modes. Even when the stimulus is varied continuously in time (Sect. 6), rather than stroboscopically switched ON and OFF, our analysis accurately identifies different forms of periodic solutions. By exploiting the fact that adaptation acts on a much slower timescale than the membrane time course of neurons, we can derive several relationships between physiologically meaningful model parameters and the behavior of the competitive neural network. 


\section{Neural Competition Model with Time-Dependent Stimuli}

We analyze a competitive neural network that is driven by intermittent stimuli. A similar model was derived in Laing and Chow (2002) from a spiking model with synaptic depression and spike frequency adaptation, reflecting deterministic dynamics of perceptual rivalry, including most of Levelt's propositions. We depart from previous studies (Noest et al. 2007; Brascamp et al. 2009) by allowing the contrast of either or both stimuli to vary in time. Therefore, we have a more general system of nonlinear, nonautonomous differential equations

$$
\begin{gathered}
\dot{u}_{L}(t)=-u_{L}(t)+H\left(\alpha u_{L}(t)-\beta u_{R}(t)-a_{L}(t)+I_{L}(t)\right), \\
\dot{u}_{R}(t)=-u_{R}(t)+H\left(\alpha u_{R}(t)-\beta u_{L}(t)-a_{R}(t)+I_{R}(t)\right), \\
\tau \dot{a}_{L}(t)=-a_{L}(t)+\phi H\left(\alpha u_{L}(t)-\beta u_{R}(t)-a_{L}(t)+I_{L}(t)\right), \\
\tau \dot{a}_{R}(t)=-a_{R}(t)+\phi H\left(\alpha u_{R}(t)-\beta u_{L}(t)-a_{R}(t)+I_{R}(t)\right),
\end{gathered}
$$

where time-dependent inputs take the form

$$
I_{j}(t)=\Delta I \cdot I(t), \quad j=L, R,
$$

and constant inputs are simply $I_{j}(t)=I_{j}(j=L, R)$ for all $t$.

Here, $u_{L}$ and $u_{R}$ represent the mean firing rate of the left and the right eye populations, respectively, with excitatory connection strength $\alpha$ and cross-inhibitory connection strength $\beta$, evolving according to (1a) and (1b). The slow negative feedback process responsible for dominance switches is spike frequency adaptation, whose variables are defined in (1c) and (1d) with strength $\phi$ and time constant $\tau$, and we assume $\tau \gg 1$ for our analysis. Our analysis could proceed similarly for system that also includes synaptic depression with sufficiently slow timescale (Laing and Chow 2002). However, adaptation alone can capture qualitative behaviors of perceptual rivalry and affords us a more transparent analysis.

The gain function $H$, which converts inputs to each neural population to an output firing rate, is taken to be a Heaviside function (Amari 1977; Laing and Chow 2002)

$$
H(x)=\left\{\begin{array}{l}
1: x \geq 0 \\
0: x<0
\end{array}\right.
$$

This idealization is often used in neural field models, to ease analytical studies of their dynamics. Seely and Chow recently studied the effects of a variety of firing rate functions upon dominance durations in related mutual inhibitory models (Seely and Chow 2011). We have also performed numerical simulations of our model (1a)-(1d) for a smooth sigmoidal firing rate function (not shown), and they correspond to the results we present here for sufficiently high gain as in Kilpatrick and Bressloff (2010).

The maximal value of the time-dependent inputs to each neural population will be $\Delta I$ and the minimum will be zero. For the majority of our analysis, we include time-dependence using the discontinuous step input

$$
I(t)=H\left(\sin \left(\frac{\pi t}{T_{I}}\right)\right)
$$


since experiments that motivate this study usually employ time-dependent stimuli whose contrast changes sharply in time (Leopold et al. 2002; Noest et al. 2007). However, we also make novel predictions regarding time-dependent changes of smoothlyvarying perceptual inputs

$$
I(t)=\frac{1}{2}\left(\sin \left(\frac{\pi t}{T_{I}}\right)+1\right),
$$

for which we can carry out some analysis determining periodic solutions that result in the network (1a)-(1d) in Sect. 6.

The model we use examines the effects that various time-dependent ambiguous stimuli have on the networks that are neural substrates of perceptual rivalry. In particular, the psychophysical experiments suggested by this theoretical work would be best carried out for binocular rivalry specifically. This is because stimuli that invoke other forms of perceptual rivalry, such as the Necker cube (Orbach et al. 1963) or the rotating sphere (Noest et al. 2007), cannot have the contrast of only a single percept altered so easily. Therefore, we consider the indices $j=L$ and $j=R$ as corresponding to neural populations encoding inputs to the left and right eye. We also note that rivalrous oscillations are known to be quite noisy (Brascamp et al. 2006), so our results should be interpreted as the mean of dominance times, influenced by the parameters of an underlying adaptive process (Lankheet 2006; Pearson and Brascamp 2008; van Ee 2011). Conceivably, we could also include noise in this model and formulate dominance switches as a first passage time problem (Moreno-Bote et al. 2007).

Analysis of our model departs from recent work on interrupted ambiguous stimuli in a few ways. First, we extensively analyze the effects of varying the strength of an input to a single population and find a fundamental mode of behavior, which we call phase-locking. Second, we use singular perturbation theory to perform a fast/slow analysis of the oscillatory states of (1a)-(1d) and predict many different complex types of behavior due to time-dependent stimuli. Finally, the terms that we use to represent spike frequency adaptation are more physiologically realistic than those presented in the models of Noest et al. (2007), Brascamp et al. (2009). The form of spike frequency adaptation included in (1a)-(1d) was derived from a spiking network in Laing and Chow (2002) and arises from an averaging calculation for a spiking neuron with a hyperpolarizing current in Benda and Herz (2003).

\section{Constant Input}

In this section, we examine some of the behaviors of the network (1a)-(1d) in the presence of constant input so that $I_{j}(t)=I_{j}$ for $j=L, R$. This will give simplified examples of the approach we use to calculate parameter region boundaries of complex behaviors in Sects. 4 and 5. In Laing and Chow (2002), a fast/slow analysis was used to approximate the relationship between dominance times and model parameters in the case of an escape mechanism. Here, we examine formulae for the release mechanism and switching brought about by combinations of release and escape.

The model (1a)-(1d) has several equilibria, as discussed by Laing and Chow (2002), depending upon the choice of parameters. When $I_{L}, I_{R}<0$, the equilibrium $u_{L}=u_{R}=0$ exists, referred to as both $O F F$. When $\alpha-\beta+I_{L}>\phi$ and 
$\alpha-\beta+I_{R}>\phi$, an equilibrium where $u_{L}=u_{R}=1$ exists, referred to as both $O N$. When $\alpha+I_{j}>\phi$ and $I_{k}-\beta<0$ (where $j=L$ or $R$, and $k \neq j$ ), an equilibrium where $u_{j}=1$ and $u_{k}=0$ exists, referred to as a winner take all (WTA) solution. Psychophysically, this corresponds to indefinite dominance of a single percept. When no equilibria exist, the system (1a)-(1d) supports oscillations, which can be generated via either release or escape (Shpiro et al. 2007). Release occurs for lower strength inputs and results in oscillations whose dominance times increase as the input is increased. In a complementary way, escape occurs for higher strength inputs and associated dominance times decrease as input strength increases, matching Levelt proposition (iv) (Levelt 1965; Shpiro et al. 2007; Curtu et al. 2008; Kilpatrick and Bressloff 2010; Seely and Chow 2011). We derive formulae for dominance times in the case of release. Despite the relations not corresponding with Levelt proposition (iv), the analysis will be an instructive prequel to our study of timedependent inputs, where we see both release and escape mechanisms.

We exploit the fact that adaptation acts more slowly $(\tau \gg 1)$ than population activity, so $u_{j} \approx 0$ or 1 for $j=L, R$, except when a switch in dominance occurs. This decouples the differential equations for $u_{j}$ from those for $a_{j}$, everywhere except the boundary layers, allowing us to understand the system (1a)-(1d) by analyzing the slow subsystem

$$
\begin{aligned}
u_{L}(t) & =H\left(\alpha u_{L}(t)-\beta u_{R}(t)-a_{L}(t)+I_{L}\right), \\
u_{R}(t) & =H\left(\alpha u_{R}(t)-\beta u_{L}(t)-a_{R}(t)+I_{R}\right), \\
\tau \dot{a}_{L}(t) & =-a_{L}(t)+\phi H_{L}, \quad \tau \dot{a}_{R}(t)=-a_{R}(t)+\phi H_{R},
\end{aligned}
$$

where $H_{j}=1$ if the $j$ th population is $\mathrm{ON}$ and $H_{j}=0$ if the $j$ th population is OFF $(j=L, R)$. We will use a fast/slow convention extensively throughout this work to draw insightful connections between the model's parameters and behaviors.

\subsection{Release and Escape Rivalry}

We proceed by assuming oscillations where a switch is generated by the release of the suppressed population. Beginning with the initial condition $u_{L}\left(0^{+}\right) \approx 1$ and $u_{R}\left(0^{+}\right) \approx 0$ immediately after a switch in dominance, the left population remains dominant for a period of time $T_{L}$. The switch in dominance occurs when the left population input falls below zero at $I_{L}+\alpha-a_{L}\left(T_{L}\right)=0$ (as in Fig. 3a). Solving (5) for the left population's adaptation variable yields

$$
a_{L}=\phi-\left(\phi-a_{L}(0)\right) e^{-t / \tau}, \quad t \in\left(0, T_{L}\right) .
$$

We can also solve (5) to compute $a_{L}$ in the case that the left population is suppressed

$$
a_{L}=a_{L}\left(T_{L}\right) e^{\left(T_{L}-t\right) / \tau}, \quad t \in\left(T_{L}, T_{L}+T_{R}\right) .
$$

Using the threshold condition for left and right release, we have

$$
a_{L}\left(T_{L}\right)=I_{L}+\alpha, \quad a_{L}\left(T_{L}+T_{R}\right)=\left(I_{L}+\alpha\right) e^{-T_{R} / \tau} .
$$


(a)

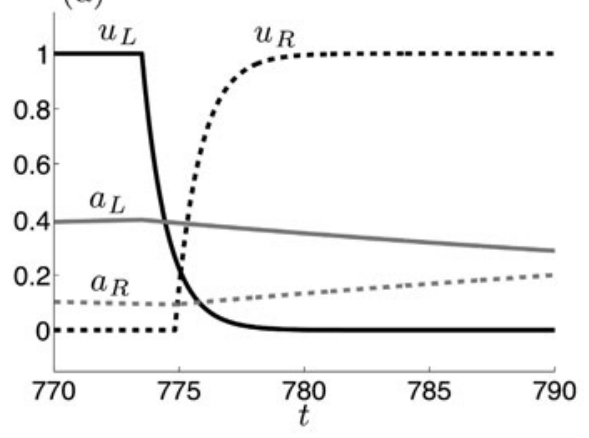

(b)

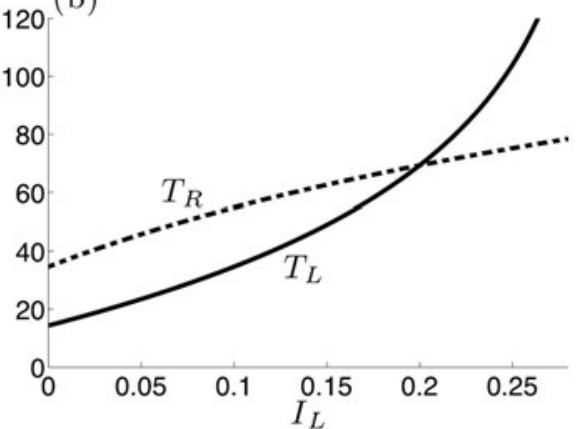

Fig. 3 Rivalry oscillations occurring due to release mechanism. (a) Plots of all model (1a)-(1d) variables for $I_{L}(t)=I_{R}(t)=0.2$, during release of the left population. Notice the boundary layer formed by the fast relaxation dynamics of $u_{L}, u_{R}$. (b) Dominance times for the left (solid) and right (dashed) populations when the right input is fixed at $I_{R}(t)=0.2$ and $I_{L}(t)=I_{L}$ is constant in time. Other parameters are $\alpha=0.2, \beta=0.5, \phi=0.5, \tau=50$

Assuming periodicity of both variables, we find

$$
\begin{aligned}
& I_{L}+\alpha=\phi-\left(\phi-\left(I_{L}+\alpha\right) e^{-T_{R} / \tau}\right) e^{-T_{L} / \tau}, \\
& I_{R}+\alpha=\phi-\left(\phi-\left(I_{R}+\alpha\right) e^{-T_{L} / \tau}\right) e^{-T_{R} / \tau} .
\end{aligned}
$$

Solving these two equations, we obtain explicit formulae for the dominance times

$$
\begin{aligned}
& T_{L}=\tau \ln \left(\frac{I_{R}+\alpha}{\phi-I_{L}-\alpha}\right), \\
& T_{R}=\tau \ln \left(\frac{I_{L}+\alpha}{\phi-I_{R}-\alpha}\right) .
\end{aligned}
$$

Foreshadowing our bifurcation analysis for time-dependent inputs, we also note that the input parameter region for which release rivalry exists is given by the inequalities $0<I_{L, R}<\phi-\alpha$ and $\phi-2 \alpha<I_{L}+I_{R}$. We plot the dominance times as a function of the left input in Fig. 3b. Notice, dominance times increase with input strength, contrary to the assertions of Levelt's proposition (iv). Since release occurs for lower contrast inputs, this may suggest that mutual inhibitory network descriptions of perceptual rivalry are only valid for sufficiently high contrast inputs (Shpiro et al. 2007).

When rivalrous oscillations are generated by an escape mechanism (see Fig. 4a), dominance times decrease as input strength increases. To compare with (6) and (7), we also present the formulae previously derived for dominance times generated by escape (Laing and Chow 2002)

$$
\begin{aligned}
& T_{L}=\tau \ln \left(\frac{\beta+\phi-I_{L}}{I_{R}-\beta}\right), \\
& T_{R}=\tau \ln \left(\frac{\beta+\phi-I_{R}}{I_{L}-\beta}\right),
\end{aligned}
$$



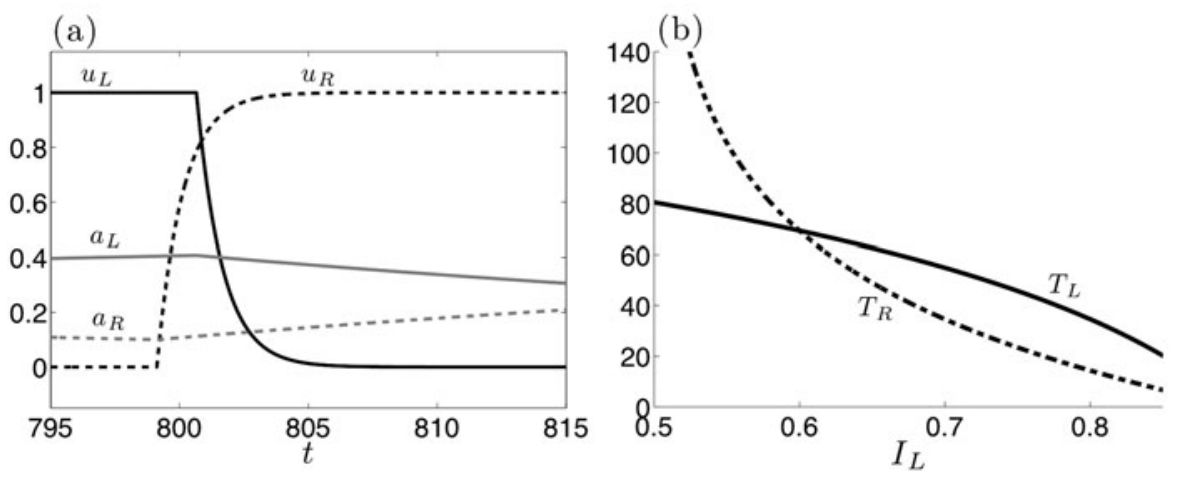

Fig. 4 Rivalry oscillations occurring due to escape mechanism. (a) Plots of all model (1a)-(1d) variables for $I_{L}(t)=I_{R}(t)=0.6$, during the escape of right population. (b) Dominance times for the left (solid) and right (dashed) populations when the right input is fixed at $I_{R}(t)=0.6$ and $I_{L}(t)=I_{L}$ is constant in time. Other parameters are $\alpha=0.2, \beta=0.5, \phi=0.5, \tau=50$

when inputs satisfy $\beta<I_{L, R}<\beta+\phi$ and $I_{L}+I_{R}<2 \beta+\phi$. While dominance times for escape oscillations depend upon the cross-inhibitory strength $\beta$, those for release oscillations depend upon self-excitatory strength $\alpha$. Dominance times decrease as input strength is increased (Fig. 4b), so escape oscillations better correspond behaviorally to Levelt's propositions (Levelt 1965; Shpiro et al. 2007; Seely and Chow 2011). This suggests that neural substrates of perceptual rivalry may depend more on the strength of cross-inhibitory connections (Blake 1989).

\subsection{Single-Population Oscillations}

We now describe solutions whereby a single population switches between being ON and OFF, regardless of the state of the other population. There is evidence that the brain's visual areas can experience hysteresis between stereopsis and binocular rivalry, which suggests complex dynamics beyond rivalry are possible (Fender and Julesz 1967; Suzuki and Grabowecky 2002; Buckthought et al. 2008). Since visual pathways for binocular rivalry and stereoscopic vision appear to be interwoven (Harrad et al. 1994), it would be valuable to see how percepts like the fusion of visual inputs are reconciled with rivalrous perception.

First, we study dynamics where the right population is ON indefinitely, and the left population switches between being ON and OFF (see Fig. 5a). We refer to the $\mathrm{ON}$ and OFF dominance times as $T_{N}$ and $T_{F}$. Assuming the left population turns ON at $t=0$, the left adaptation variable satisfies

$$
a_{L}\left(T_{N}\right)=\frac{\phi\left(1-e^{-T_{N} / \tau}\right)}{1-e^{\left(-T_{F}-T_{N}\right) / \tau}}, \quad a_{L}(0)=\frac{\phi\left(e^{-T_{F} / \tau}-e^{-T_{N}-T_{F}}\right)}{1-e^{\left(-T_{N}-T_{F}\right) / \tau}} .
$$

Upon applying the threshold conditions $a_{L}\left(T_{N}\right)=I_{L}+\alpha-\beta$ and $a_{L}(0)=I_{L}-\beta$, we have formulae for the $\mathrm{ON}$ and $\mathrm{OFF}$ time of the left population

$$
T_{N}=\tau \ln \left(\frac{\beta+\phi-I_{L}}{\beta+\phi-I_{L}-\alpha}\right),
$$



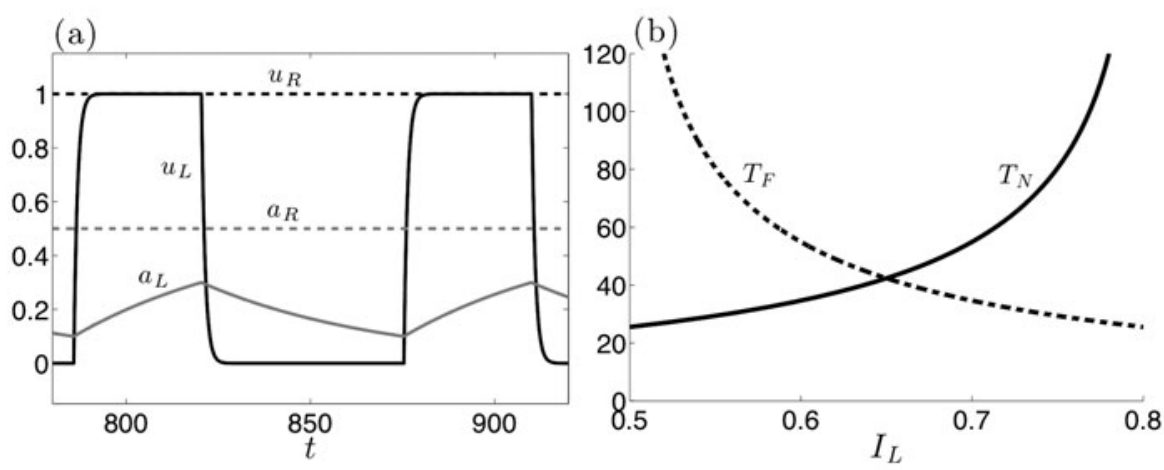

Fig. 5 Left population oscillates while the right population remains ON. (a) Plots of all model (1a)-(1d) variables with constant inputs $I_{R}=1.1$ and $I_{L}=0.6$. (b) Plots of amount of time left population spends $\mathrm{ON}\left(T_{N}\right)$ and $\mathrm{OFF}\left(T_{F}\right)$, where $I_{R}=1.1$ and $I_{L}(t)=I_{L}$ is constant in time. Other parameters are $\alpha=0.2$, $\beta=0.5, \tau=50$, and $\phi=0.5$

$$
T_{F}=\tau \ln \left(\frac{I_{L}+\alpha-\beta}{I_{L}-\beta}\right)
$$

For this state to exist, input parameters must satisfy $\beta<I_{L}<\beta+\phi-\alpha$ and $I_{R}>$ $\beta+\phi-\alpha$. We plot the dominance and suppression times as a function of $I_{L}$ in Fig. $5 \mathrm{~b}$. As the left input increases, the OFF time decreases and the ON time increases.

In a similar way, we examine the $\mathrm{ON}$ and OFF times of a left population oscillation when the right population is OFF indefinitely to find

$$
\begin{aligned}
T_{N} & =\tau \ln \left(\frac{\phi-I_{L}}{\phi-I_{L}-\alpha}\right), \\
T_{F} & =\tau \ln \left(\frac{I_{L}+\alpha}{I_{L}}\right),
\end{aligned}
$$

where input parameters must satisfy $0<I_{L}<\phi-\alpha$ and $I_{R}<0$. Notice, there is no dependence upon the cross-inhibition parameter $\beta$ in this case. The solutions we find suggest that a single population's adaptation may have the capacity to switch percepts ON and OFF, without the need for cross-inhibition. Network oscillations between sole dominance of a single percept and a fusion state may arise when one input's contrast is substantially stronger than the other.

Finally, we show a case where the left and right populations become active in succession, followed by a period in which both populations are OFF (see Fig. 6a). To solve for the switching times, we refer to the left and right populations' dominance times as $T_{L}$ and $T_{R}$ and the time both are simultaneously OFF as $T_{F}$. We can solve for approximations of the adaptation variables and derive an implicit system for the switching times

$$
I_{R}+\alpha=\phi-\left(\phi-\frac{\phi\left(e^{\left(-T_{F}-T_{L}\right) / \tau}-e^{\left(-T_{L}-T_{F}-T_{R}\right) / \tau}\right)}{1-e^{\left(-T_{L}-T_{F}-T_{R}\right) / \tau}}\right) e^{-T_{R} / \tau},
$$


(a)

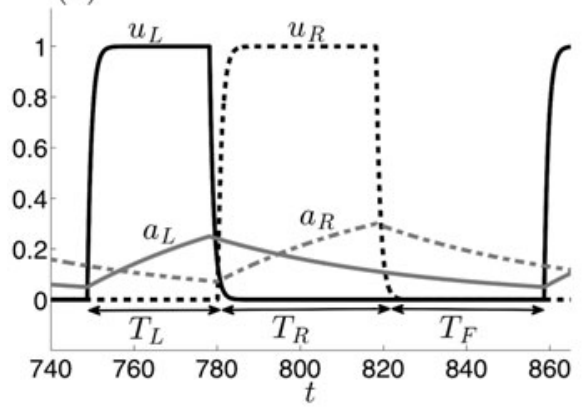

(b)

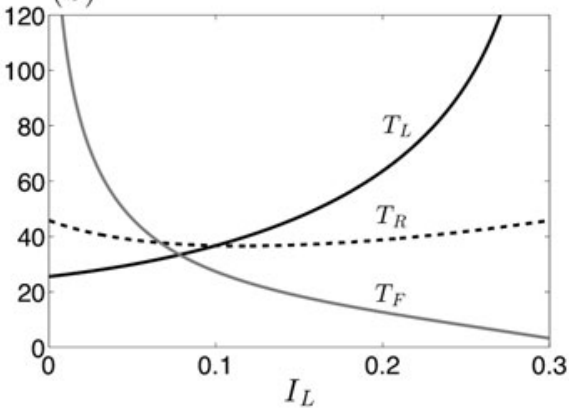

Fig. 6 Rivalrous oscillation accompanied by a punctuating OFF period. Orbit has a total period of $T_{L}+T_{R}+T_{F}$. (a) Plots of all model (1a)-(1d) variables for $I_{L}=0.05$ and $I_{R}=0.1$. (b) Plot of the switching times for $I_{R}(t)=0.1$ and $I_{L}(t)=I_{L}$ is constant in time. Other parameters are $\alpha=0.2, \beta=0.5$, $\tau=50$, and $\phi=0.5$

$$
\begin{aligned}
I_{L} & =\left(\frac{\phi\left(1-e^{\left(-T_{L}\right) / \tau}\right)}{1-e^{\left(-T_{F}-T_{R}\right) / \tau}}\right) e^{\left(-T_{R}-T_{F}\right) / \tau}, \\
I_{L}+\alpha & =\phi-\left(\phi-\frac{\phi\left(e^{\left(-T_{F}-T_{R}\right) / \tau}-e^{\left(-T_{L}-T_{F}-T_{R}\right) / \tau}\right)}{1-e^{\left(-T_{L}-T_{F}-T_{R}\right) / \tau}}\right) e^{-T_{L} / \tau} .
\end{aligned}
$$

Dominance times determined using numerical root finding are plotted as functions of the left input in Fig. 6b. For this behavior to exist, inputs need to be substantially weak. A complementary behavior exists where rivalry combines with both populations being ON simultaneously for substantially strong inputs.

Thus, we show alternative dynamics to pure rivalry can exist, even when inputs are constant in (1a)-(1d). Perturbative fast/slow timescale approximations adequately predict the switching times of these more intricate behaviors. Similar techniques to those used for static inputs can also be applied to cases where inputs are varied in time.

\section{Time-Variation in Single Input}

We now analyze the effects of varying one population's input in time $I_{L}(t)$, while keeping the other input $I_{R}$ constant. Assuming the step input (3), we can perform a fast/slow analysis on the model (1a)-(1d) to identify different modes of behavior. Our analysis allows several new qualitative predictions regarding the effects of varying the contrast of a single eye's input in binocular rivalry experiments.

\subsection{Phase-Locked Behavior}

We first characterize a mode of behavior we call the phase-locked state (see Fig. 7a). When the left input is $\mathrm{ON}\left(I_{L}(t)=\Delta I\right)$, we assume the left population is ON and the right is OFF. When the left input is $\mathrm{OFF}\left(I_{L}(t)=0\right)$, the right population is $\mathrm{ON}$ and the left is OFF. As defined by the input function, durations of either percept are $T_{I}$ 
(a)

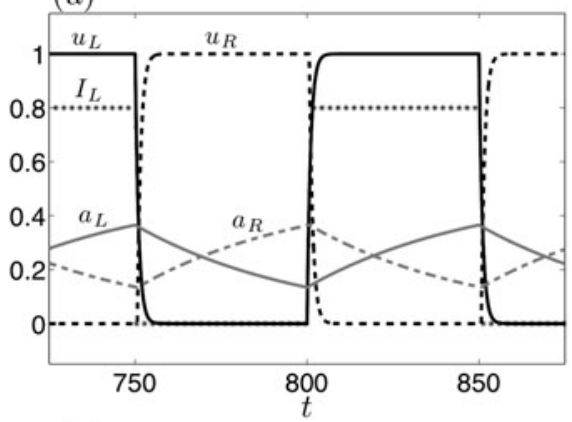

(c)

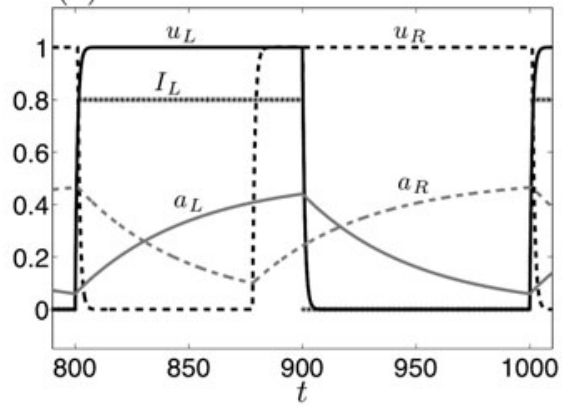

(b)

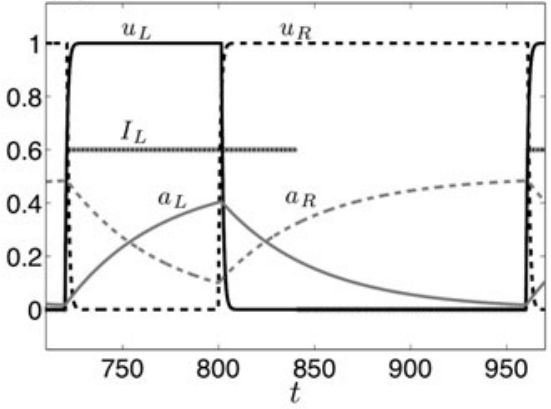

(d)

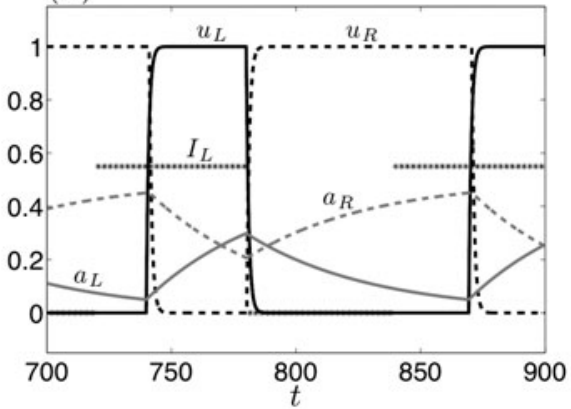

Fig. 7 Various periodic solutions to the system (1a)-(1d) with a single time dependent step input (3). (a) Phase-locking $(\mathrm{Ph})$ of left population to stimulus $\left(\Delta I=0.8\right.$ and $\left.T_{I}=50\right)$. (b) Early escape of the right population (RE) to a switch in dominance $\left(\Delta I=0.6\right.$ and $\left.T_{I}=120\right)$. (c) Escape of right population to a fusion (EF) ON state $\left(\Delta I=0.8\right.$ and $\left.T_{I}=100\right)$. (d) Left population fails to switch ON (LF) immediately with stimulus $\left(\Delta I=0.55\right.$ and $\left.T_{I}=60\right)$. Other parameters are $\alpha=0.2, \beta=0.5, \tau=50, \phi=0.5, I_{R}=0.6$

each. Since switching is entirely induced by the time-dependence of the input here, adaptation variables do not affect the time duration of population activity. However, there are inequalities involving the adaptation variables that must be satisfied in order for the phase-locked state to exist.

We now derive the set of inequalities that must be satisfied in the phase-locked state. Assuming that the system starts at $t=0$ with the input $I_{L}(t)$ just switching ON, so the left population $u_{L} \approx 1$ and the right population $u_{R} \approx 0$, we have the two inequalities

$$
\alpha+\Delta I>a_{L}(t) \quad \text { and } \quad-\beta+I_{R}<a_{R}(t), \quad t \in\left(0, T_{I}\right),
$$

involving the inputs to the left and right population. Since switching must be entirely accomplished by the time-dependent input, we require no release occurs for the left population

$$
\alpha+\Delta I>a_{L}\left(T_{I}\right)=\phi-\left(\phi-a_{L}(0)\right) e^{-T_{I} / \tau},
$$

and no escape occurs for the right population

$$
I_{R}-\beta<a_{R}\left(T_{I}\right)=a_{R}(0) e^{-T_{I} / \tau} .
$$


Now we derive two similar inequalities for the second part of the oscillation, when $I_{L}(t)=0$. At the beginning, the left population should switch OFF

$$
\alpha<a_{L}\left(T_{I}\right)=\phi-\left(\phi-a_{L}(0)\right) e^{-T_{I} / \tau},
$$

and the right population should switch $\mathrm{ON}$ immediately after

$$
I_{R}>a_{R}\left(T_{I}\right)=a_{R}(0) e^{-T_{I} / \tau} .
$$

For the time period from $t=T_{I}$ to $t=2 T_{I}$, the states of each population must not change and the left population will certainly not escape since

$$
-\beta<0<a_{L}(t), \quad t \in\left(T_{I}, 2 T_{I}\right),
$$

is always true, and the right population must not release

$$
\alpha+I_{R}>a_{R}(t), \quad t \in\left(T_{I}, 2 T_{I}\right) .
$$

Since both populations remain $\mathrm{ON}$ and OFF for equal lengths of time, phaseshifted one half-period from one another, their adaptation variables have the same time-course, up to a half-period shift. Thus, we note periodicity $a_{L}(0)=a_{L}\left(2 T_{I}\right)$ and the value of $a_{L}$ at the end of the ON and OFF phases by

$$
\begin{gathered}
a_{L}\left(T_{I}\right)=\phi-\left(\phi-a_{L}(0)\right) e^{-T_{I} / \tau}, \\
a_{L}\left(2 T_{I}\right)=\phi e^{-T_{I} / \tau}-\left(\phi-a_{L}(0)\right) e^{-2 T_{I} / \tau} .
\end{gathered}
$$

We can solve for the initial values of the left and right adaptation variables

$$
a_{L}(0)=\frac{\phi\left(e^{-T_{I} / \tau}-e^{-2 T_{I} / \tau}\right)}{1-e^{-2 T_{I} / \tau}}, \quad a_{R}(0)=\frac{\phi\left(1-e^{-T_{I} / \tau}\right)}{1-e^{-2 T_{I} / \tau}} .
$$

Including these expressions in the inequalities derived and simplifying, we have seven restrictions on the parameter space in which phase-locking can occur, based on requirements of left $(\mathrm{L})$ and right $(\mathrm{R})$ population dynamics:

(Ph-i) No release of $\mathrm{L}: \quad \alpha+\Delta I>\frac{\phi}{1+e^{-T_{I} / \tau}}$,

(Ph-ii) No escape of R: $I_{R}-\beta<\frac{\phi}{1+e^{T_{I} / \tau}}$,

(Ph-iii) L switching OFF: $\quad \alpha<\frac{\phi}{1+e^{-T_{I} / \tau}}$,

(Ph-iv) R switching ON: $I_{R}>\frac{\phi}{1+e^{T_{I} / \tau}}$,

(Ph-v) No release of R: $\quad I_{R}+\alpha>\frac{\phi}{1+e^{-T_{I} / \tau}}$,

(Ph-vi) L switching ON: $\quad-\beta+\Delta I>\frac{\phi}{1+e^{T_{I} / \tau}}$, 
(Ph-vii) R switching OFF: $\quad I_{R}+\alpha-\beta<\frac{\phi}{1+e^{-T_{I} / \tau}}$.

For weak alternating inputs $\Delta I$, the left population will not switch ON every time the input $I_{L}$ switches to a high state. For long half-periods $T_{I}$, the right population will start to release or escape during the period of assumed right or left dominance. By simply varying the form of input to the network, we can study new dynamics of population activity that emerges once phase-locking breaks down. These periodic solutions involve the release and escape of either population, rather than simple inputinduced switching ON and OFF. It is also possible for the network to fail to lock to the time-varying stimulus in the $1: 1$ fashion that is clear in phase-locking, leading to other $1: n$ mode-locked states. Further analysis allows us to relate stimulus parameters to complex behaviors of the competitive neural network.

\subsection{Escape by Right Population}

For long stimulus periods, the left population's dominance time can be cut short by the escape of the right population (see Fig. 7b). Analysis follows along the same lines as the derivation of phase-locked parameter inequalities, but now we derive an expression for the right population's escape time $T_{R}$. Here, the left population switches ON at $t=0$ but will be switched OFF at $t \approx T_{R}$ where

$$
I_{R}-\beta=a_{R}\left(T_{R}\right)=\frac{\phi\left(e^{-T_{R} / \tau}-e^{-2 T_{I} / \tau}\right)}{1-e^{-2 T_{I} / \tau}},
$$

meaning we can solve explicitly for the right population's escape time

$$
T_{R}=\tau \log \left(\frac{\phi}{\left(I_{R}-\beta\right)\left(1-e^{-2 T_{I} / \tau}\right)+\phi e^{-2 T_{I} / \tau}}\right) .
$$

After a similar series of calculations to the phase-locked case, we can express the inequalities that constrain parameters for right escape (RE) to exist:

(RE-i) No Release of L: $\Delta I+\alpha>\beta-I_{R}+\phi$,

(RE-ii) Escape of R: $\quad I_{R}-\beta>\frac{\phi}{1+e^{T_{I} / \tau}}$,

(RE-iii) L switches OFF: $\Delta I+\alpha-\beta<\beta-I_{R}+\phi$,

(RE-iv) No Release of R: $I_{R}+\alpha>\frac{\phi e^{2 T_{I} / \tau}\left(I_{R}-\beta\right)}{\left(I_{R}-\beta\right)\left(e^{2 T_{I} / \tau}-1\right)+\phi}$,

(RE-v) L switches ON: $\Delta I-\beta>\frac{\phi\left(\beta-I_{R}+\phi\right)}{\left(I_{R}-\beta\right)\left(e^{2 T_{I} / \tau}-1\right)+\phi}$,

(RE-vi) R switches OFF: $\quad I_{R}+\alpha-\beta<\frac{\phi\left(I_{R}-\beta\right) e^{2 T_{I} / \tau}}{\left(I_{R}-\beta\right)\left(e^{2 T_{I} / \tau}-1\right)+\phi}$.

We use these inequalities in generating Fig. 9 at the end of this section, exhibiting the partitioning of parameter space into different types of behavior. 


\subsection{Escape to Fusion}

A further variation on simple phase-locked behavior includes a period in which both populations are either ON or OFF at the same time. Psychophysically, this would correspond to the dominance of both (fusion of two) or neither percepts for a noticeable time window. Such behavior may be a time-dependent version of the hysteresis observed in experiments where the orientation difference between binocular images is slowly changed (Buckthought et al. 2008).

We show a behavior where the left population is still locked to the input of the stimulus, but the right population switches ON (see Fig. 7c). Here, the left population starts out dominant and the right population suppressed. Then, the right population switches ON and a fusion period begins at the time $T_{R}$ given by the expression (10). At $t=T_{I}$, the left population switches OFF along with the stimulus. Finally, at $t=$ $2 T_{I}$, the left population turns $\mathrm{ON}$ and suppresses the right population, completing the periodic solution. Using a fast/slow analysis, we derive the following inequalities:

(EF-i) No Release of L: $\quad \Delta I+\alpha-\beta>\frac{\phi}{1+e^{-T_{I} / \tau}}$,

(EF-ii) No Release of $\mathrm{R}$ while $\mathrm{L}$ is $\mathrm{ON}$ :

$$
I_{R}+\alpha-\beta>\frac{\phi\left[\left(I_{R}-\beta\right)\left(e^{2 T_{I} / \tau}+e^{T_{I} / \tau}-1\right)+\phi-\phi e^{T_{I} / \tau}\right]}{\left(I_{R}-\beta\right)\left(e^{2 T_{I} / \tau}-1\right)+\phi},
$$

(EF-iii) L switches OFF: $\quad \alpha-\beta<\frac{\phi}{1+e^{-T_{I} / \tau}}$,

(EF-iv) No Release of $\mathrm{R}$ while L is OFF:

$$
I_{R}+\alpha>\frac{\phi\left(I_{R}-\beta\right) e^{2 T_{I} / \tau}}{\left(I_{R}-\beta\right)\left(e^{2 T_{I} / \tau}-1\right)+\phi},
$$

(EF-v) L switches ON: $\Delta I-\beta>\frac{\phi}{1+e^{T_{I} / \tau}}$,

(EF-vi) R switches OFF: $\quad I_{R}+\alpha-\beta<\frac{\phi\left(I_{R}-\beta\right) e^{2 T_{I} / \tau}}{\left(I_{R}-\beta\right)\left(e^{2 T_{I} / \tau}-1\right)+\phi}$.

Combinations of fusion and rivalry in periodic solutions of competitive neural networks have seldom been studied, but there is experimental evidence for these types of dynamics. Periodic perturbations of spatially structured binocular rivalry stimuli have been shown to create periods of "mixed dominance" where the two percepts fuse together in between one percept and the other being dominant (Kang et al. 2009), suggesting visual streams for stereoscopy and rivalry may be the same.

\subsection{Left Fails to Switch ON}

It is also possible that the left population may cease to lock to the stimulus state by failing to switch ON when the stimulus switches ON. The right population remains in dominance for longer than a half stimulus period $T_{I}$ (Fig. 7d). The inequality (Phvi) breaks down here, and there are different dominance times for the left and right 
(a)

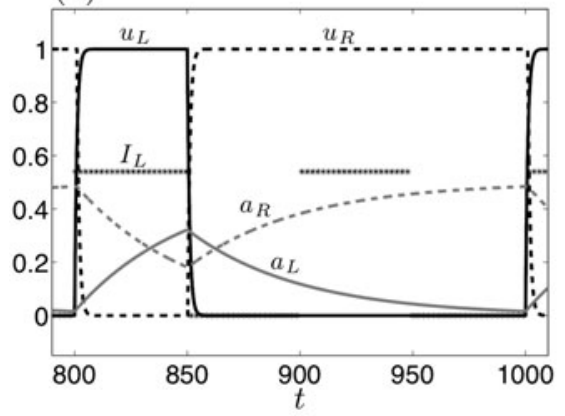

(b)

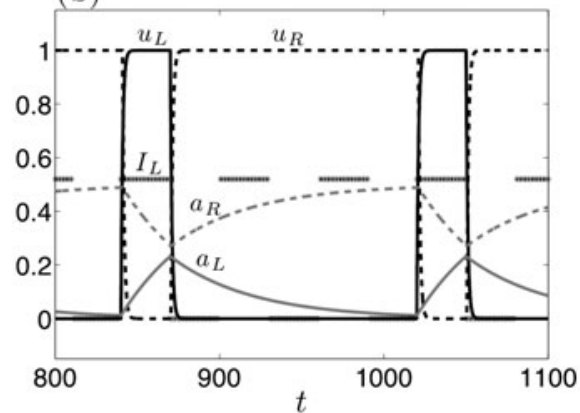

Fig. 8 Cycle skipping generates $1: n$ mode-locked solutions in network (1a)-(1d) with left input interrupted in time where (a) $n=2$ for $\Delta I=0.54$ and $T_{I}=50$ and (b) $n=3$ for $\Delta I=0.52$ and $T_{I}=30$. Other parameters are $\alpha=0.2, \beta=0.5, \phi=0.5, \tau=50, I_{R}=0.6$

population. The left population escapes at $t=T_{L}$, so the right population stays on for $T_{I}+T_{L}$ and the left stays $\mathrm{ON}$ for $T_{I}-T_{L}$. Computing the form of the adaptation variables, we produce the following set of inequalities, bounding parameter space in which this behavior exists:

(LF-i) No Release of R: $I_{R}+\alpha>\beta-\Delta I+\phi$,

(LF-ii) R switches OFF: $I_{R}+\alpha-\beta<\beta-\Delta I+\phi$,

(LF-iii) No Release of L: $\quad \Delta I+\alpha>\frac{\phi(\Delta I-\beta) e^{2 T_{I} / \tau}}{(\Delta I-\beta)\left(e^{2 T_{I} / \tau}-1\right)+\phi}$,

(LF-iv) L switches OFF: $\quad \alpha<\frac{\phi(\Delta I-\beta) e^{2 T_{I} / \tau}}{(\Delta I-\beta)\left(e^{2 T_{I} / \tau}-1\right)+\phi}$,

(LF-v) R switches ON: $\quad I_{R}>\frac{\phi(\phi-\Delta I+\beta)}{(\Delta I-\beta)\left(e^{2 T_{I} / \tau}-1\right)+\phi}$.

Failure of the left population to switch ON with the stimulus indicates the system can slip from the stimulus time course, though there is still a $1: 1$ correspondence between the network period and the stimulus period. Now, we examine how $1: n$ mode-locked solutions arise when the left population fails to switch $\mathrm{ON}$ during every other stimulus-ON epoch.

\subsection{Mode-Locking Through Cycle Skipping}

We now explicitly compute the parameter space boundaries of $1: 2$ mode-locking that arise through cycle skipping (see Fig. 8a). For weak input amplitudes $\Delta I$ or short time scales $T_{I}$, the left population's adaptation does not recover enough during stimulus interruptions for phase-locking to occur. Once the stimulus is turned ON again, the left population does not turn $\mathrm{ON}$ at all. As behavior moves away from pure phaselocking, 1:2 mode-locking can arise as a solution where the network completes one period for every two stimulus periods. In the case of $1: 2$ mode-locking, where each 
Fig. 9 Partitioning of single time-variant input parameter space $\left(T_{I}, \Delta I\right)$ into different dynamical behaviors: phase-locked; R escape/fusion; fusion followed by left $\mathrm{OFF}$ (fus/L OFF); right escape; left switching ON fails; $(1: 2)$ mode-locked solutions; $1: n$ mode-locked solutions ( $\mathrm{m})$; and right $\mathrm{ON}$ left $\mathrm{OFF}$ indefinitely. Other parameters are $\alpha=0.2$, $\beta=0.5, \phi=0.5, \tau=50$, $I_{R}=0.6$

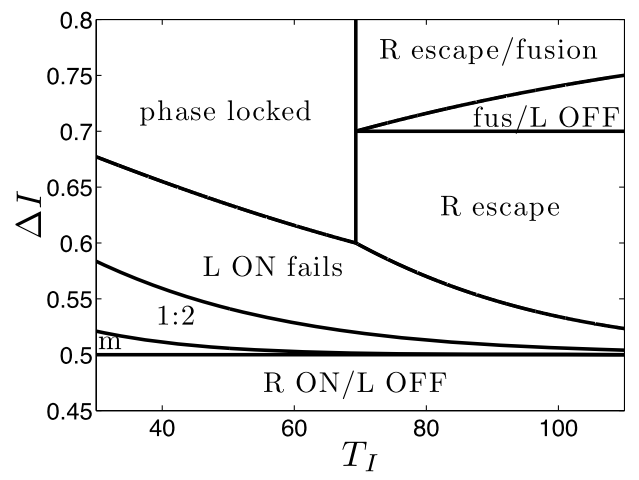

left $\mathrm{ON}$ epoch lasts $T_{I}$, the right population is $\mathrm{ON}$ for a duration of $3 T_{I}$, suppressing the left population in the meantime. There are other $1: 2$ mode-locked solutions, but we summarize the parameter space boundaries associated with this straightforward case here. Parameter inequalities associated with this solution are:

$$
\begin{aligned}
& \text { (1 : 2-i) No Release of L: } \quad \Delta I+\alpha>\frac{\phi}{\left(1+e^{-T_{I} / \tau}\right)\left(1+e^{-2 T_{I} / \tau}\right)}, \\
& \text { (1 : 2-ii) L switches OFF: } \quad \alpha<\frac{\phi}{\left(1+e^{-T_{I} / \tau}\right)\left(1+e^{-2 T_{I} / \tau}\right)}, \\
& \left(1: 2-\text { iii) No Release of R: } \quad I_{R}+\alpha>\frac{\phi\left(1-e^{-3 T_{I} / \tau}\right)}{1-e^{-4 T_{I} / \tau}},\right. \\
& \left(1: 2 \text {-iv) No Escape of L: } \quad \Delta I-\beta<\frac{\phi}{\left(1+e^{-T_{I} / \tau}\right)\left(1+e^{2 T_{I} / \tau}\right)},\right. \\
& (1: 2-v) \text { L switches ON: } \quad \Delta I-\beta>\frac{\phi}{\left(1+e^{T_{I} / \tau}\right)\left(1+e^{2 T_{I} / \tau}\right)} .
\end{aligned}
$$

Beyond $1: 2$ mode-locked solutions, if the input $\Delta I$ is even weaker, or the input timescale $T_{I}$ shorter, there are $1: n$ mode-locked solutions where $n>2$ for $n \in \mathbb{Z}^{+}$. For example, in Fig. 8b, a $1: 3$ mode-locked solution is shown, where the left population turns $\mathrm{ON}$ every third stimulus presentation. We could similarly extend the inequalities presented here to provide bounds on the existence of $1: n$ mode-locked solutions for $n>2$. We summarize these behaviors in our partitioning of parameter space by noting where these multiple cycle skips appear.

\subsection{Partition of Parameter Space}

Now, we can use our accumulated bounds to display the resulting partition of parameter space $\left(T_{I}, \Delta I\right)$ into a variety of different behaviors. We show this partitioning in Fig. 9. The primary behavior of phase-locking occurs for sufficiently high contrast $\Delta I$ and brief enough duration $T_{I}$. This prediction is intuitive, since an observer would likely discern the higher contrast of two percepts prior to the dynamics of adaptation 
generating a switch (Blake and Logothetis 2002). For a long timescale of periodic image presentation, the right population (percept) appears through either early fusion or dominance while the left image is still ON. For weak stimuli, the left percept does not switch ON immediately with its input. As the stimulus amplitude is weakened further or the input period is lessened, this effect becomes more pronounced. This can even lead to the left percept skipping one or multiple stimulus epochs as shown in the region of $1: n$ mode-locked solutions, (1:2) and (m) respectively.

Many qualitatively new behaviors emerge as we temporally vary the left input that could correspond to complex perceptual states that emerge when the stimulus is presented intermittently over time to an observer. We now proceed to examine some more qualitatively different behaviors that emerge from the system when we vary both inputs over time.

\section{Time-Variation in Both Inputs}

Several authors have examined the effect of simultaneously switching both inputs ON and OFF in a competitive neural network (Noest et al. 2007; Brascamp et al. 2009; Gigante et al. 2009), in an attempt to understand how interrupted stimuli allow a single percept to remain in dominance for longer (Orbach et al. 1963; Leopold et al. 2002; Blake et al. 2003; Chen and He 2004). They did not probe more complex dynamics, which we study here. Exploiting a fast/slow analysis, we can partition parameter space, in a similar way to the single input varied case of Sect. 4, into different dynamical behaviors. We assume both inputs vary in the same way, according to the stepwise input (3).

\subsection{Phase-Locked ON/OFF Oscillation}

The simplest possible periodic solution is one where both populations turn $\mathrm{ON}$ and OFF together, with the input (see Fig. 10a). Activity variables should then be identical, as should the adaptation variables. Performing a fast/slow analysis, we arrive at the same expression for both adaptation variables here as in the phase-locked case for the single varied input

$$
a_{j}(0)=a_{j}\left(2 T_{I}\right)=\frac{\phi}{1+e^{T_{I} / \tau}} \quad \text { and } \quad a_{j}\left(T_{I}\right)=\frac{\phi}{1+e^{-T_{I} / \tau}}, \quad j=L, R .
$$

Now, we require that neither population escapes or releases and that they are only switched ON and OFF by changes in the input's state. These requirements, along with the formulae for the adaptation variables yield four inequalities, restricting parameter space for this solution:

$$
\begin{aligned}
& \text { (OF-i) No Release: } \Delta I+\alpha-\beta>\frac{\phi}{1+e^{-T_{I} / \tau}}, \\
& \text { (OF-ii) Switching OFF: } \quad \alpha-\beta<\frac{\phi}{1+e^{-T_{I} / \tau}},
\end{aligned}
$$


(a)

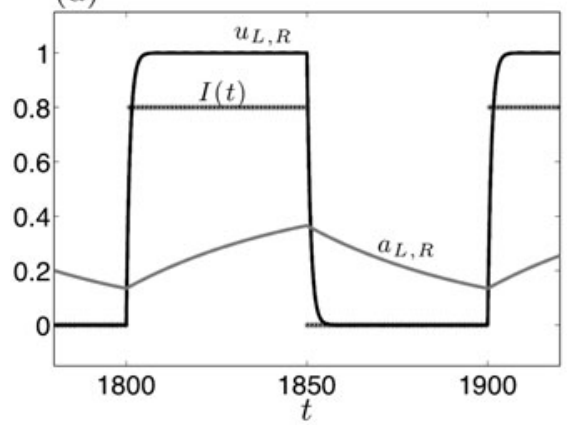

(c)

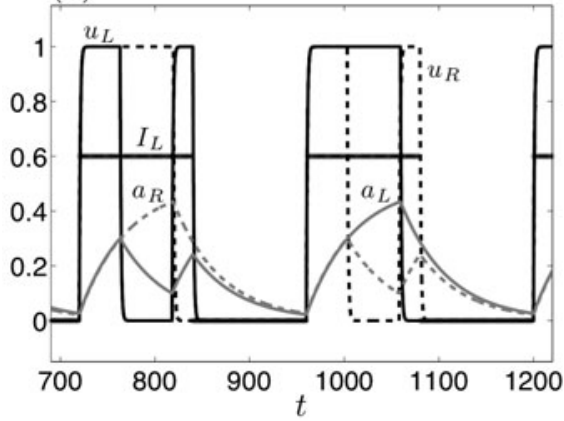

(b)

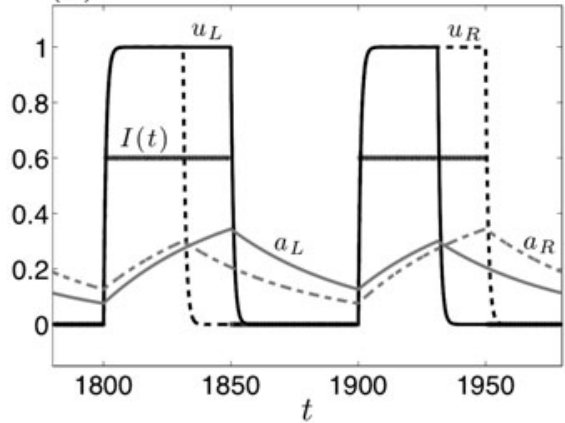

(d)

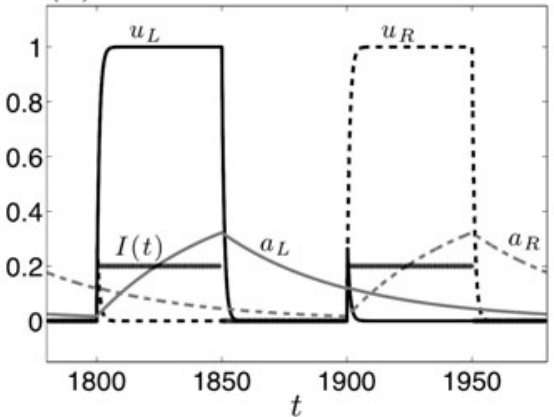

Fig. 10 Various periodic behaviors of the system (1a)-(1d) when both inputs $I_{L}(t), I_{R}(t)$ are the periodic step (3). (a) Phase-locked ON/OFF oscillation where both populations lock to time-dependent stimulus ( $\Delta I=0.8$ and $T_{I}=50$ ). (b) Rivalry/fusion oscillation where both populations turn ON with the stimulus, and there is an alternation in the population that releases each stimulus presentation $(\Delta I=0.6$ and $T_{I}=50$ ). (c) Dominance switching where there are multiple alternations in dominance with each stimulus presentation $\left(\Delta I=0.6\right.$ and $\left.T_{I}=120\right)$. (d) Rivalry where dominance switches after each stimulus interruption $\left(\Delta I=0.2\right.$ and $\left.T_{I}=50\right)$. Other parameters are $\alpha=0.2, \beta=0.5, \phi=0.5, \tau=50$

(OF-iii) No Escape: $\quad 0<\frac{\phi}{1+e^{T_{I} / \tau}}$,

(OF-iv) Switching ON: $\Delta I>\frac{\phi}{1+e^{T_{I} / \tau}}$.

Inequality (iii) is always satisfied, since $\phi$ must be positive. Existence of this phaselocked ON/OFF oscillation is then determined by three inequalities that restrict parameter space. We will use these to build a diagram of parameter space at the conclusion of this section (Fig. 11). Now, we turn to studying rivalrous behavior.

\subsection{Rivalry/Fusion}

To study more intricate dynamics, different from that found in previous work, we examine half-periods $T_{I}$ commensurate with the adaptation time constant $\tau$. Note, we also restrict the OFF period of the stimulus to be the same as the ON period, which is unlike protocols that generate stabilization, since they usually require a longer 
Fig. 11 Partitioning of the parameter space of input parameters $\left(T_{I}, \Delta I\right)$ into various behaviors, when both populations are driven by time-dependent input. The derivation of a boundary is given in the subsection corresponding to the behavior it borders. Other parameters are $\alpha=0.2, \beta=0.5$, $\tau=50$, and $\phi=0.5$

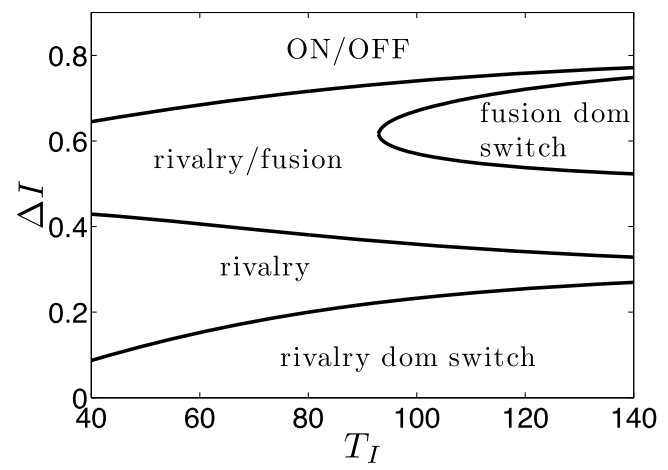

OFF period (Leopold et al. 2002; Blake et al. 2003; Noest et al. 2007). The first periodic solution we examine is one where both populations are $\mathrm{ON}$ for a window of time, a fusion state, followed by one population releasing (see Fig. 10b), due to a breakdown in inequality (OF-i). Then, there is an alternation in the solely dominant population from one stimulus period to the next, making this a period-two oscillation. This can occur for inputs with long half-periods $T_{I}$ or weak amplitudes $\Delta I$. Due to the underlying reflection symmetry of the system, we can assume identical switching times for either population.

Thus, we derive the set of inequalities that must be satisfied in this fusion/rivalry state. Assuming the system starts at $t=0$ with the input $I(t)$ just switching ON, causing both populations to turn $\mathrm{ON}$, we have the following inequalities

$$
\Delta I>a_{L}(0) \text { and } \alpha-\beta+\Delta I>a_{R}(0),
$$

assuming the left population will switch ON slightly before the right. Now, we pick the right population as the first to release at the time $T_{S}$. This generates the inequality and equality

$$
\alpha-\beta+\Delta I>a_{L}\left(T_{S}\right) \quad \text { and } \quad \alpha-\beta+\Delta I=a_{R}\left(T_{S}\right) .
$$

In addition, we require that the right and left populations remain subthreshold and superthreshold, respectively, while the input is ON so that

$$
\alpha+\Delta I>a_{L}\left(T_{I}\right) \text { and }-\beta+\Delta I<a_{R}\left(T_{I}\right) .
$$

When the input switches OFF, there is the additional requirement that the left population switches OFF, $\alpha<a_{L}\left(T_{I}\right)$. A similar series of events occurs once the stimulus turns $\mathrm{ON}$ again, with the roles of the left and right populations reversed. This yields a set of conditions that does not add new information to the parameter space restriction, due to the system's symmetry. Based on this series of events, we can derive expressions for both adaptation variables, which have the same time course up to a $2 T_{I}$ time shift. We can thus specify both adaptation variables by solving the piecewise-linear differential equations, imposing periodic boundary conditions $\left(a_{j}(0)=a_{j}\left(4 T_{I}\right) ; j=L, R\right)$, and the relation $a_{R}(t)=a_{L}\left(t+2 T_{I}\right)$. Plugging these values into our set of inequalities, we have: 
(RF-i) L (R) switches ON

$$
\Delta I>\frac{\phi e^{-2 T_{I} / \tau}\left(1-e^{-T_{I} / \tau}+e^{-2 T_{I} / \tau}\right)}{\frac{\phi+\beta-\alpha-\Delta I}{\alpha-\beta+\Delta I}+e^{-4 T_{I} / \tau}},
$$

(RF-ii) R (L) switches ON

$$
\alpha-\beta+\Delta I>\frac{\phi \frac{\phi+\beta-\alpha-\Delta I}{\alpha-\beta+\Delta I}\left(e^{-T_{I} / \tau}-e^{-2 T_{I} / \tau}\right)+\phi e^{-4 T_{I} / \tau}}{\frac{\phi+\beta-\alpha-\Delta I}{\alpha-\beta+\Delta I}+e^{-4 T_{I} / \tau}},
$$

(RF-iii) No release of L (R)

$$
\alpha-\beta+\Delta I>\frac{(\alpha-\beta+\Delta I)\left(1+e^{-2 T_{I} / \tau}-e^{-3 T_{I} / \tau}\right)+\phi\left(e^{-T_{I} / \tau}-e^{-2 T_{I} / \tau}\right)}{1-e^{-T_{I} / \tau}+e^{-2 T_{I} / \tau}},
$$

(RF-iv) No release of $L(R)$

$$
\alpha+\Delta I>\frac{\phi \frac{\phi+\beta-\alpha-\Delta I}{\alpha-\beta+\Delta I}\left(1-e^{-T_{I} / \tau}\right)+\phi e^{-3 T_{I} / \tau}}{\frac{\phi+\beta-\alpha-\Delta I}{\alpha-\beta+\Delta I}+e^{-4 T_{I} / \tau}},
$$

(RF-v) No escape of R (L)

$$
-\beta+\Delta I<\frac{\phi e^{-T_{I} / \tau}\left(1-e^{-T_{I} / \tau}+e^{-2 T_{I} / \tau}\right)}{\frac{\phi+\beta-\alpha-\Delta I}{\alpha-\beta+\Delta I}+e^{-4 T_{I} / \tau}},
$$

(RF-vi) L (R) switches OFF

$$
\alpha<\frac{\phi \frac{\phi+\beta-\alpha-\Delta I}{\alpha-\beta+\Delta I}\left(1-e^{-T_{I} / \tau}\right)+\phi e^{-3 T_{I} / \tau}}{\frac{\phi+\beta-\alpha-\Delta I}{\alpha-\beta+\Delta I}+e^{-4 T_{I} / \tau}} .
$$

Thus, we have six nonlinear inequalities that represent the boundary in parameter space for the existence of this solution, the combination of fusion and rivalry.

\subsection{Rivalry}

For weak inputs, there is no fusion ON state in the periodic solution. In this case, there can be a pure rivalry state where populations alternate dominance with each $\mathrm{ON}$ period of the stimulus (see Fig. 10d). To derive bounds for this state, we consider the case where the left population is switched $\mathrm{ON}$ at $t=0$, forcing the right population to be suppressed, generating the inequalities

$$
\Delta I>a_{L}(0) \text { and } \alpha-\beta+\Delta I<a_{R}(0) .
$$

The last inequality arises because the right population may switch $\mathrm{ON}$ for a brief period (much shorter than $\tau$ ), but the left population will win after this transient phase. After this first ON period, the left and right populations must have stayed ON 
and OFF, respectively, but the left should immediately shut OFF, when the stimulus does

$$
\alpha+\Delta I>a_{L}\left(T_{I}\right), \quad \Delta I<a_{R}\left(T_{I}\right), \text { and } \alpha<a_{L}\left(T_{I}\right) .
$$

After the OFF stage of the stimulus, we now require the right population be switched $\mathrm{ON}$ at $t=2 T_{I}$, forcing the left population to be suppressed, which generates an analogous set of inequalities with the roles of the right and left population reversed. By imposing periodic boundary conditions, we solve for the values of the adaptation variables, affording us the following collection of inequalities:

$$
\begin{aligned}
& \text { (Riv-i) L (R) switches ON: } \quad \Delta I>\frac{\phi}{\left(1+e^{T_{I} / \tau}\right)\left(1+e^{2 T_{I} / \tau}\right)}, \\
& \text { (Riv-ii) R (L) is suppressed: } \quad \alpha-\beta+\Delta I<\frac{\phi}{\left(1+e^{T_{I} / \tau}\right)\left(1+e^{-2 T_{I} / \tau}\right)}, \\
& \text { (Riv-iii) No Release of L (R): } \quad \alpha+\Delta I>\frac{\phi}{\left(1+e^{-T_{I} / \tau}\right)\left(1+e^{-2 T_{I} / \tau}\right)}, \\
& \text { (Riv-iv) No Escape of R (L): } \quad \Delta I-\beta<\frac{\phi}{\left(1+e^{-T_{I} / \tau}\right)\left(1+e^{2 T_{I} / \tau}\right)}, \\
& \text { (Riv-v) L (R) switches OFF: } \quad \alpha<\frac{\phi}{\left(1+e^{-T_{I} / \tau}\right)\left(1+e^{-2 T_{I} / \tau}\right)} .
\end{aligned}
$$

The system's reflection symmetry allows us to only concern ourselves with the set of inequalities of a single ON/OFF epoch.

\subsection{Dominance Switching}

There are a few different periodic solutions where dominance switches multiple times during a single stimulus presentation epoch. It is also possible to derive parameter boundaries for these types of behavior. However, the resulting formulae are usually more complicated than those involved in the inequalities presented to this point. In addition, these more intricate responses to time-dependent stimuli may be more difficult to detect in experiments, since dominance switching is noisy (Blake and Logothetis 2002; Brascamp et al. 2006). For these reasons, we omit the analysis in this work.

Nonetheless, we do show one such behavior in Fig. 10c. In this case, both populations switch $\mathrm{ON}$ with the stimulus. Following this, either population may release to allow the other population dominance. It later escapes suppression to provide a switch in dominance. The particular population that releases initially then alternates with each stimulus presentation. We label this behavior as "fusion dom switch" in our partition of parameter space in Fig. 11. There are other behaviors where dominance switches multiple times in an epoch but no fusion occurs, which we call "rivalry dom switch."

\subsection{Partition of Parameter Space}

We have characterized several of the behaviors present when both inputs to a competitive neural network are varied in time. In particular, we have produced boundaries in 
the space of input parameters $T_{I}$ and $\Delta I$ that give excellent approximations of where these behaviors exist. We show this partitioning in Fig. 11. Several principals can be extracted from this diagram. First, fairly strong inputs bypass the rivalry process and lead to a fusion state, even in time-dependent stimuli, overriding the effects of adaptation. As inputs are weakened, adaptation can take effect. For long stimulus periods, each stimulus ON epoch can host switches in dominance that occur through escape. As inputs are weakened further, stimulus ON epochs no longer lead to any fusion at all, and at most one population is ON at any given time. The "rivalry" state we find leads to a different percept existing for the duration of the stimulus epoch, alternating with each presentation. If inputs are weak enough, dominance switches begin to occur during epochs via release.

The results of our analysis suggest a few hypotheses that could be tested in perceptual experiments. As the contrast of an interrupted ambiguous stimulus is reduced, one would expect an observer to perceive transitions from fusion to rivalrous behavior. In addition, as the time scale of intermittently presented stimuli is varied, one should expect to see more dominance switching per stimulus epoch, but only a single dominance period for sufficiently fast interruption times. Thus, exploring bifurcations in models of rivalry with time-varied stimuli provides a number of reasonable predictions that could be tested in experiments on binocular rivalry. Now, we proceed to study the effects of smooth time-varying stimuli on network dynamics.

\section{Sinusoidal Input}

In this section, we explore the effects of a continuous, rather than a piecewise constant input. It is not straightforward to develop explicit inequalities that describe the boundaries in parameter space between different periodic solutions. Nonetheless, we can focus on the mode-locking of solutions in the system (1a)-(1d) when a single population's input is sinusoidal in time (4). Thus, we can classify how well a competitive network locks its representation of binocular stimuli to input with continuously varying contrast. Thinking of the entire competitive neural network as an oscillator, we can frame this work in the context of previous studies of mode-locking in neuronal networks (Coombes and Bressloff 1999). When both populations' inputs are sinusoidal in time (4), we examine the existence of a fusion-rivalry solution. This persists over a wide range of parameter values and may be an interesting sequence of percepts to examine in future experiments.

\subsection{Single Time-Varied Input}

First, we examine 1:1 mode-locking, which we refer to as phase-locking in our analysis of the system driven by the step input (3). As shown in Fig. 12a, this is a $2 T_{I}$-periodic solution where the left population switches $\mathrm{ON}$ when its input $I_{L}$ rises, suppressing the right population, at a time we denote $T_{\Delta}$ such that

$$
-\beta+I_{L}\left(T_{\Delta}\right)=a_{L}\left(T_{\Delta}\right)
$$


(a)

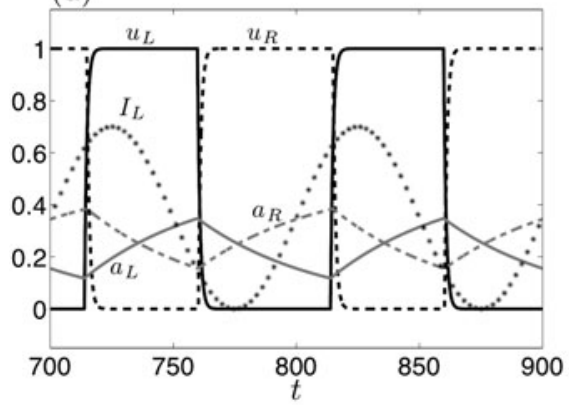

(b)

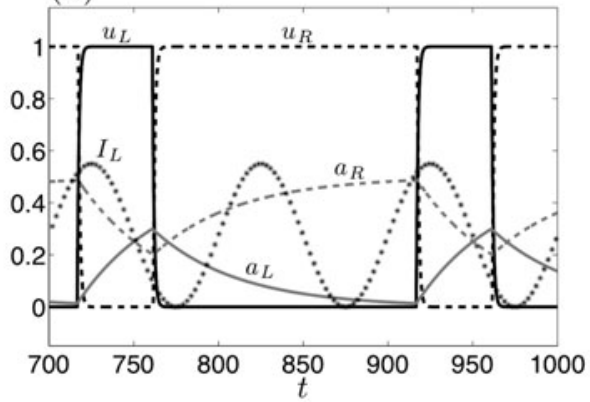

Fig. 12 Mode-locked solutions in the network (1a)-(1d) when a single population receives sinusoidally varied input (4). (a) $1: 1$ mode-locked solution for input parameters $\Delta I=0.7$ and $T_{I}=50$. (b) $1: 2$ mode-locked solution for input parameters $\Delta I=0.55$ and $T_{I}=50$. Other parameters are $\alpha=0.2, \beta=0.5$, $\phi=0.5, \tau=50, I_{R}=0.5$

The left population will then switch OFF after a dominance time duration $T_{L}$, once the input $I_{L}$ decreases to a sufficiently weak value so that

$$
\alpha+I_{L}\left(T_{\Delta}\right)=a_{L}\left(T_{\Delta}+T_{L}\right),
$$

allowing the right population to subsequently switch ON. Determining the resultant evolution of the left population's adaptation variable $a_{L}$ and employing the functional form of the sinusoidal input, (11) and (12) become

$$
\begin{aligned}
& \frac{\Delta I}{2}\left(\sin \left(\frac{\pi T_{\Delta}}{T_{I}}\right)+1\right)-\beta=\frac{\phi\left(e^{\left(-2 T_{I}+T_{L}\right) / \tau}-e^{-2 T_{I} / \tau}\right)}{1-e^{-2 T_{I} / \tau}}, \\
& \frac{\Delta I}{2}\left(\sin \left(\frac{\pi\left(T_{\Delta}+T_{L}\right)}{T_{I}}\right)+1\right)+\alpha=\frac{\phi\left(1-e^{-T_{L} / \tau}\right)}{1-e^{-2 T_{I} / \tau}} .
\end{aligned}
$$

We shall use these implicit expressions for the dominance time of the left population to generate a bifurcation diagram revealing period-adding that can occur in this system (see Fig. 13a).

Beyond this bifurcation, through period-adding, the subsequent periodic solution that lies in this forced system is a $1: 2$ mode-locked solution (as shown in Fig. 12b). In this case, the forms of the equations for the left population switching $\mathrm{ON}$ and OFF are similar

$$
\begin{aligned}
I_{L}\left(T_{\Delta}\right)-\beta & =a_{L}\left(T_{\Delta}\right), \\
I_{L}\left(T_{\Delta}+T_{L}\right)+\alpha & =a_{L}\left(T_{\Delta}+T_{L}\right) .
\end{aligned}
$$

However, the adaptation variable of the left population $a_{L}$ has a longer duration to recover between $\mathrm{ON}$ epochs. Solving for this function and evaluating the input, (14) and (15) become

$$
\frac{\Delta I}{2}\left(\sin \left(\frac{\pi T_{\Delta}}{T_{I}}\right)+1\right)-\beta=\frac{\phi\left(e^{\left(-4 T_{I}+T_{L}\right) / \tau}-e^{-4 T_{I} / \tau}\right)}{1-e^{-4 T_{I} / \tau}},
$$



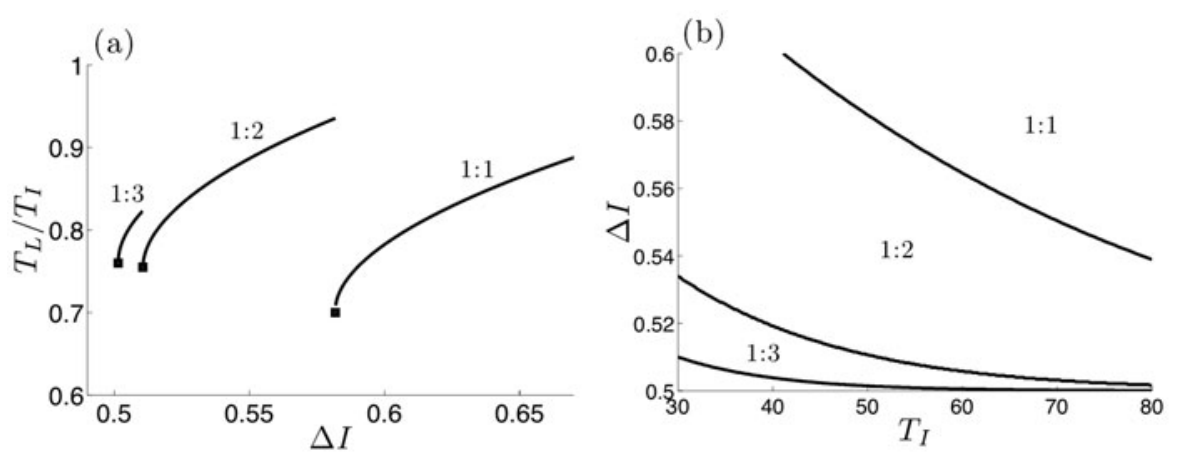

Fig. 13 (a) Left population dominance time's fraction of the stimulus half-period $T_{L} / T_{I}$ plotted versus the stimulus amplitude $\Delta I$ for different $1: n$ mode-locking to stimulus half-period $T_{I}=50$. Each solution branch is annihilated in a period-adding bifurcation (squares). (b) Period-adding bifurcation boundaries plotted in input parameter space $\left(T_{I}, \Delta I\right)$. Different $1: n$ mode-locking regions are labeled. Unlabeled region includes $1: n$ locking for $n \in \mathbb{Z}^{+}$and $n \geq 4$. Other parameters are $\alpha=0.2, \beta=0.5, \phi=0.5$, $\tau=50, I_{R}=0.5$

$$
\frac{\Delta I}{2}\left(\sin \left(\frac{\pi\left(T_{\Delta}+T_{L}\right)}{T_{I}}\right)+1\right)+\alpha=\frac{\phi\left(1-e^{-T_{L} / \tau}\right)}{1-e^{-4 T_{I} / \tau}} .
$$

This system of equations can also support two solutions that annihilate in a periodadding bifurcation for sufficiently fast or weak input. In general, the implicit set of equations that must be satisfied for a $1: n$ mode-locked solution to exist is

$$
\begin{aligned}
& \frac{\Delta I}{2}\left(\sin \left(\frac{\pi T_{\Delta}}{T_{I}}\right)+1\right)-\beta=\frac{\phi\left(e^{\left(-2 n T_{I}+T_{L}\right) / \tau}-e^{-2 n T_{I} / \tau}\right)}{1-e^{-2 n T_{I} / \tau}}, \\
& \frac{\Delta I}{2}\left(\sin \left(\frac{\pi\left(T_{\Delta}+T_{L}\right)}{T_{I}}\right)+1\right)+\alpha=\frac{\phi\left(1-e^{-T_{L} / \tau}\right)}{1-e^{-2 n T_{I} / \tau}} .
\end{aligned}
$$

As the stimulus intensity $\Delta I$ is reduced, the system undergoes numerous transitions from $1: n$ locked states to $1:(n+1)$ locked states. We visualize these in Fig. 13a by plotting the ratio $T_{L} / T_{I}$ against the associated $\Delta I$ value, revealing the period-adding bifurcation structure. Notice, there is only one branch for each $1: n$ mode-locked solution, even though the shape of the branch might suggest that it is the stable branch of a saddle/node pair. This is because what would be the corresponding saddle solution does not satisfy the following inequalities, requiring the left and right populations stay ON and OFF for their specified durations of a $1: n$ mode-locked solution:

$$
\begin{aligned}
& \alpha+I_{L}(t)>a_{L}(t): t \in\left(T_{\Delta}, T_{\Delta}+T_{L}\right), \\
& -\beta+I_{R}<a_{R}\left(T_{\Delta}+T_{L}\right), \quad I_{R}>a_{R}\left(T_{\Delta}+T_{L}\right), \\
& -\beta+I_{L}(t)<a_{L}(t): t \in\left(T_{\Delta}+T_{L}, T_{\Delta}+2 n T_{I}\right), \\
& \alpha+I_{R}>a_{R}\left(T_{\Delta}\right), \quad \alpha-\beta+I_{R}<a_{R}\left(T_{\Delta}\right) .
\end{aligned}
$$


We can track the value $\Delta I$ at each period-adding bifurcation point as a function of $T_{I}$ by simply identifying where the set of nonlinear equations (16) and (17) ceases to have a solution. Doing so, we show regions in parameter space where particular $1: n$ locked solutions exist in Fig. 13b. The area of parameter space in which $1: n$ mode-locked solutions exist appears to decrease as $n$ increases. As the amplitude and timescale of the stimulus decreases, it is more difficult for the network to lock to the input signal since there is less time for the adaptation variable to recover in between successive high stimulus states.

\subsection{Time-Varied Inputs in Both Populations}

Finally, we consider the effects of smoothly varying the contrast of both inputs in time. Once again, we use a sinusoidal input function (4) that is identical for both populations. A common solution that arises in this case is a combination of fusion and rivalry. Here, a single population turns ON, then the other switches ON, followed by the first population switching OFF, and finally the second population switches OFF (see Fig. 14a). Notice, due to the underlying symmetry of the system, the ordering of the events can be flipped to yield a similar solution for the same set of parameters (see Fig. 14b).

This solution is determined by four values: $T_{\Delta}$, the shift of the first ON population from the input timing; $T_{L}$, the sole dominance time of the left population; $T_{U}$, the amount of time both populations are ON simultaneously (fusion); and $T_{R}$, the sole dominance time of the right population. We can compute these values by following the order of events in a fast/slow analysis. Presuming the left population is the first to switch $\mathrm{ON}$, then the input at that time $I\left(-T_{\Delta}\right)$ must cross through the value of the left adaptation variable $a_{L}\left(-T_{\Delta}\right)$ such that $I\left(-T_{\Delta}\right)=a_{L}\left(-T_{\Delta}\right)$. In order that the right population switch $\mathrm{ON}$ to create a fusion state at $t=T_{L}-T_{\Delta}$, we require $I\left(T_{L}-T_{\Delta}\right)-\beta=a_{R}\left(T_{L}-T_{\Delta}\right)$. Then the left population will switch OFF after the amount of time $T_{U}$ over which fusion occurs, so that

$$
I\left(T_{U}+T_{L}-T_{\Delta}\right)+\alpha-\beta=a_{L}\left(T_{U}+T_{L}-T_{\Delta}\right) .
$$

(a)

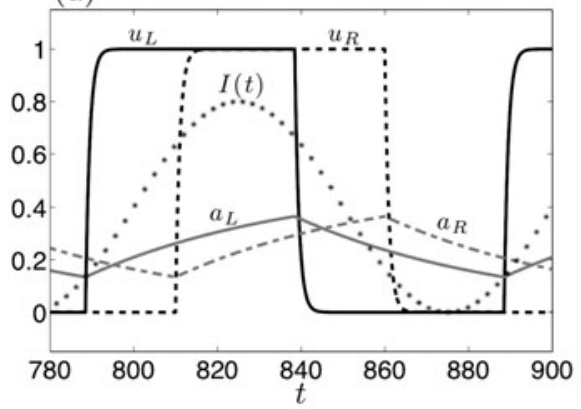

(b)

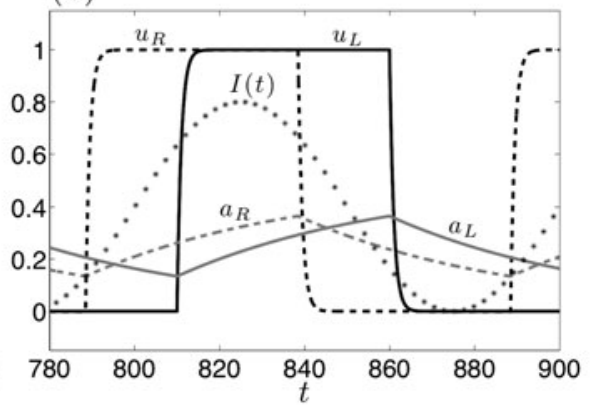

Fig. 14 Rivalry/fusion solutions in the network (1a)-(1d) when a both population receive sinusoidally varied input (4) when the (a) left and (b) right population switches ON first. Parameters are $\alpha=0.2$, $\beta=0.5, \phi=0.5, \tau=50, T_{I}, \Delta I=0.8$ 
Finally, the right population switches OFF after $T_{R}$, its sole dominance time

$$
I\left(T_{U}+T_{L}+T_{R}-T_{\Delta}\right)+\alpha=a_{R}\left(T_{U}+T_{L}+T_{R}-T_{\Delta}\right) .
$$

Upon solving for the adaptation variables and employing the sinusoidal input function (4), we arrive at the following four equations for the associated durations of each phase of the behavior

$$
\begin{aligned}
& \frac{\Delta I}{2}\left(1-\sin \left(\frac{\pi T_{\Delta}}{T_{I}}\right)\right)=\phi \frac{e^{\left(-2 T_{I}+T_{L}+T_{U}\right) / \tau}-e^{-2 T_{I} / \tau}}{1-e^{-2 T_{I} / \tau}}, \\
& \frac{\Delta I}{2}\left(\sin \left(\frac{\pi\left(T_{L}-T_{\Delta}\right)}{T_{I}}\right)+1\right)-\beta=\phi \frac{e^{\left(-2 T_{I}+T_{R}+T_{U}\right) / \tau}-e^{-2 T_{I} / \tau}}{1-e^{-2 T_{I} / \tau}}, \\
& \frac{\Delta I}{2}\left(\sin \left(\frac{\pi\left(T_{U}+T_{L}-T_{\Delta}\right)}{T_{I}}\right)+1\right)+\alpha-\beta=\phi \frac{1-e^{\left(-T_{L}-T_{U}\right) / \tau}}{1-e^{-2 T_{I} / \tau}}, \\
& \frac{\Delta I}{2}\left(\sin \left(\frac{\pi\left(T_{U}+T_{L}+T_{R}-T_{\Delta}\right)}{T_{I}}\right)+1\right)+\alpha=\phi \frac{1-e^{\left(-T_{R}-T_{U}\right) / \tau}}{1-e^{-2 T_{I} / \tau}} .
\end{aligned}
$$

We can use a numerical root finding algorithm to find the dependence of the durations of each system state upon input parameters $\Delta I$ and $T_{I}$, as shown in Fig. 15. We also impose inequality bounds (not shown), as in the case of the single varied input, to ensure that the left and right populations stay ON and OFF during the correct time domains. In Fig. 15a, we see that the normalized length of the fusion period increases with stimulus amplitude, while that of the sole dominance durations decreases. This may be related to Levelt's proposition (iv), which indicates dominance times should decrease with stimulus amplitude for constant stimuli (Levelt 1965). Interestingly, in our case, the mechanism for termination of the sole dominance times is escape for $T_{L}$ and release for $T_{R}$. Notice, in Fig. 15b, that the normalized dominance durations of each behavior phase is relatively unaffected by changes in $T_{I}$, except for the delay time.
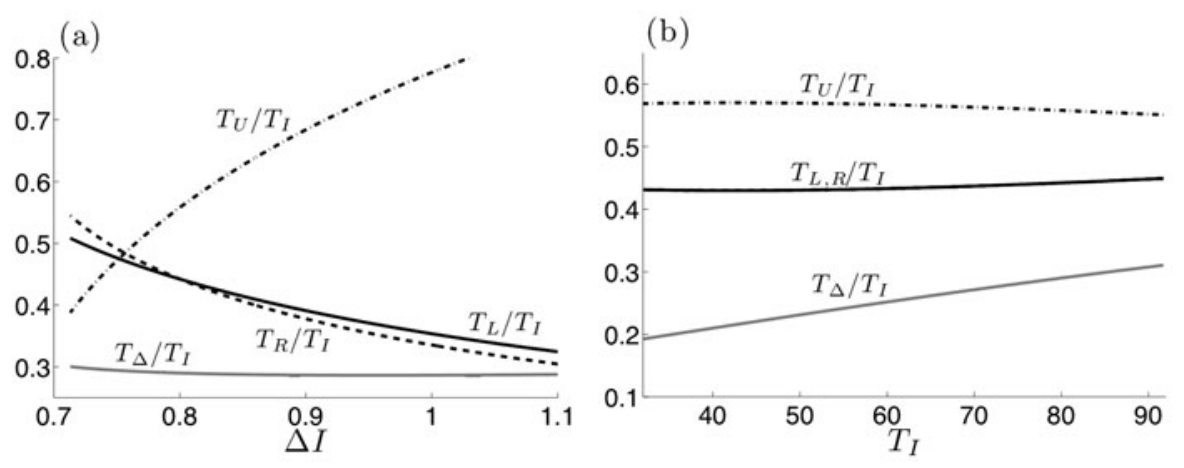

Fig. 15 Dependence of the duration of each phase of the fusion/rivalry solution on (a) the maximum amplitude $\Delta I$, for $T_{I}=80$, and (b) the timescale $T_{I}$, for $\Delta I=0.8$, of a sinusoidal input to the competitive neural network (1a)-(1d). Other parameters are $\alpha=0.2, \beta=0.5, \phi=0.5, \tau=50$ 
We have developed several testable predictions for the effects of continuously varying contrasts of binocular rivalry stimuli in time. First, we predict that there should be some region of 1:1 mode-locking in the space of stimulus parameters when the contrast of a single percept is varied in time. As the amplitude and timescale of the stimulus is reduced, networks responsible for encoding the stimulus can no longer track every period of contrast variation and cycle skipping occurs. Thus, through a period-adding bifurcation, different mode-locked behaviors can occur within the network. When both stimuli are varied identically in time, this can lead to a combination of fusion and rivalry in the activity of the network encoding the signal. Studying the duration of each phase of this periodic solution of network activity provides general relationships between the stimulus amplitude and duration of different combinations of network activation. Thus, we present novel ideas for quantifiable binocular rivalry experiments that could further assist in identifying the organization of visual processing in the brain.

\section{Discussion}

We have explored the effects of different types of time-varying stimuli in a competitive neural network with adaptation and have classified new dynamical behaviors in the context of their perceptual interpretations. Even in the case of constant stimuli, we showed oscillatory solutions aside from standard escape or release rivalry examined previously (Laing and Chow 2002; Taylor et al. 2002; Shpiro et al. 2007; Curtu et al. 2008; Kilpatrick and Bressloff 2010; Seely and Chow 2011) can arise as the contrasts of stimuli are varied. Fast/slow analysis, which exploited the slow timescale of adaptation, was the main technique we used to derive quantities related to each type of behavior. In particular, we found behaviors where a single population could switch ON and OFF, regardless of the state of the other population. We then explored a variety of novel oscillatory behaviors that arise by varying the contrast of inputs to either or both populations in time. When a single input was periodically interrupted, the associated population could lock to the state of the input for high values of $\Delta I$ and low values of the input period $T_{I}$. This phase-locked behavior ceased to exist if input parameters were substantially varied and mechanisms of escape and release created more complex dynamics. When both inputs were periodically interrupted in time, we found several combinations of rivalry and fusion behaviors. As opposed to previous modeling studies that primarily focused on percept stabilization for rapidly interrupted stimuli (Noest et al. 2007; Brascamp et al. 2009; Gigante et al. 2009), we examined complex dynamics that arise when interruptions occur on a slower timescale. As the amplitude of the stimulus was reduced there were transitions from fusion oscillations, to mixes of fusion and rivalry, to a true rivalry type behavior. Finally, we analyzed the system when inputs were sinusoidal in time. When a single population received sinusoidal input, we note the existence of a modelocked solution where the percepts alternate in dominance. A novel feature of the smooth input case is the existence of a period adding between $1: n$ mode-locked solutions. When both populations received sinusoidal input, we found a common mode of behavior was a combination of rivalry and fusion for which we could calculate the 
durations of each phase of behavior. In total, our analysis suggests that a host of novel oscillatory behaviors in competitive networks can arise when input is constant and especially time-dependent. We believe these provide a rich avenue for study in theoretical models of competitive networks (Laing and Chow 2002; Shpiro et al. 2007; Noest et al. 2007) as well as experimental studies of perceptual rivalry (Blake and Logothetis 2002; Pearson and Brascamp 2008).

Perceptual rivalry has long provided a noninvasive way of uncovering neural substrates of visual experience (Blake and Logothetis 2002). Our results suggest several directions for gauging contrast dependent mechanisms in binocular vision and comparing models of perceptual rivalry to potential data sets. The periodic interruption of a single input could be easily enacted in a binocular rivalry experiment. For high contrasts, our analysis predicts the subject should immediately perceive the interrupted stimulus, once it is turned $\mathrm{ON}$, if the OFF period is long enough. This would provide substantial support for the claim that dominance switches in perceptual rivalry are mainly governed by a slow adaptive process (Laing and Chow 2002; Lankheet 2006; van Ee 2011) rather than noise (Brascamp et al. 2006; Moreno-Bote et al. 2007). As the length of the stimulus ON period is increased, it would be interesting to see when subjects lose this locking of their perception to the phase of the input. Stimuli whose contrast varies continuously in time could also be used to quantify a relation between dominance times of each percept and stimulus amplitude. It is interesting that the dominance time of a percept increases with increasing contrast, as shown in our analysis in Sect. 6. This reverses the contrast dependence of dominance times present in constant input paradigms, where dominance time decreases as contrast increases according to Levelt proposition (iv) (Levelt 1965; Seely and Chow 2011). Future experiments could employ periodic stimuli of longer timescales that potentially lead to the combinations of fusion and rivalry seen in our analysis. This may relate to existing evidence of hysteresis between fusion and rivalry in previous experiments (Buckthought et al. 2008). Rather than simply recording dominance times, patients could indicate when the stimulus paradigm produces a mixture of rivalry and fusion in their perception.

Our results also suggest several interesting directions for future theoretical work on perceptual rivalry and competitive neural networks. In preliminary numerical simulations of a network with a smooth firing rate function and continuously varying input, we have found novel dynamical behaviors such as mixed mode oscillations (MMOs). This is not surprising, since recently Curtu has characterized MMOs in a competitive neural network model where adaptation depends linearly upon firing rate (Curtu 2010). However, studying such behavior in a nonautonomous system may offer new challenges that could be handled, perhaps, using Floquet theory. By adding noise into our model, we could examine how robust the boundaries partitioning input parameters are in the face of perturbation. In particular, the parameter region in which pure phase-locking to a single population's input may shrink, and we could quantify this decrease by formulating dominance switching as a first passage time problem (Moreno-Bote et al. 2007, 2010). Finally, synaptic depression has also been proposed as a mechanism that produces dominance switching in competitive neural networks for perceptual rivalry (Laing and Chow 2002; Taylor et al. 2002; Shpiro et al. 2007; Kilpatrick and Bressloff 2010). Analyzing a system that has this 
divisive, rather than subtractive, form of negative feedback may lead to further complex dynamics not addressed in this study.

Acknowledgements S.J. was supported by an NSF Research Experience for Undergraduates Fellowship (EMSW21-RTG 0739261). Z.P.K. is supported by an NSF Mathematical Sciences Postdoctoral Research Fellowship (DMS-1004422).

\section{References}

Abbott, L. F., Varela, J. A., Sen, K., \& Nelson, S. B. (1997). Synaptic depression and cortical gain control. Science, 275(5297), 220-224.

Amari, S.-I. (1977). Dynamics of pattern formation in lateral-inhibition type neural fields. Biol. Cybern., 27(2), 77-87.

Benda, J., \& Herz, A. V. M. (2003). A universal model for spike-frequency adaptation. Neural Comput., 15(11), 2523-2564.

Blake, R. (1989). A neural theory of binocular rivalry. Psychol. Rev., 96(1), 145-167.

Blake, R., \& Logothetis, N. (2002). Visual competition. Nat. Rev., Neurosci., 3, 1-11.

Blake, R., Sobel, K. V., \& Gilroy, L. A. (2003). Visual motion retards alternations between conflicting perceptual interpretations. Neuron, 39(5), 869-878.

Bossink, C. J., Stalmeier, P. F., \& De Weert, C. M. (1993). A test of Levelt's second proposition for binocular rivalry. Vis. Res., 33(10), 1413-1419.

Brascamp, J. W., van Ee, R., Noest, A. J., Jacobs, R. H. A. H., \& van den Berg, A. V. (2006). The time course of binocular rivalry reveals a fundamental role of noise. J. Vis., 6(11), 1244-1256.

Brascamp, J. W., Knapen, T. H. J., Kanai, R., Noest, A. J., van Ee, R., \& van den Berg, A. V. (2008). Multi-timescale perceptual history resolves visual ambiguity. PLoS ONE, 3(1), e1497.

Brascamp, J. W., Pearson, J., Blake, R., \& van den Berg, A. V. (2009). Intermittent ambiguous stimuli: implicit memory causes periodic perceptual alternations. J. Vis., 9(3), 3.1.

Buckthought, A., Kim, J., \& Wilson, H. R. (2008). Hysteresis effects in stereopsis and binocular rivalry. Vis. Res., 48(6), 819-830.

Chen, X., \& He, S. (2004). Local factors determine the stabilization of monocular ambiguous and binocular rivalry stimuli. Curr. Biol., 14(11), 1013-1017.

Coombes, S., \& Bressloff, P. C. (1999). Mode locking and Arnold tongues in integrate-and-fire neural oscillators. Phys. Rev. E, 60(2 Pt B), 2086-2096.

Curtu, R. (2010). Singular Hopf bifurcations and mixed-mode oscillations in a two-cell inhibitory neural network. Physica D, 239(9), 504-514.

Curtu, R., Shpiro, A., Rubin, N., \& Rinzel, J. (2008). Mechanisms for frequency control in neuronal competition models. SIAM J. Appl. Dyn. Syst., 7(2), 609-649.

Fender, D., \& Julesz, B. (1967). Extension of Panum's fusional area in binocularly stabilized vision. J. Opt. Soc. Am., 57(6), 819-830.

Gigante, G., Mattia, M., Braun, J., \& Del Giudice, P. (2009). Bistable perception modeled as competing stochastic integrations at two levels. PLoS Comput. Biol., 5(7), e1000430.

Harrad, R. A., McKee, S. P., Blake, R., \& Yang, Y. (1994). Binocular rivalry disrupts stereopsis. Perception, 23(1), 15-28.

Kang, M. S., Heeger, D., \& Blake, R. (2009). Periodic perturbations producing phase-locked fluctuations in visual perception. J. Vis., 9(2), 8.1.

Kilpatrick, Z. P., \& Bressloff, P. C. (2010). Binocular rivalry in a competitive neural network with synaptic depression. SIAM J. Appl. Dyn. Syst., 9(4), 1303-1347.

Klink, P. C., van Ee, R., Nijs, M. M., Brouwer, G. J., Noest, A. J., \& van Wezel, R. J. A. (2008). Early interactions between neuronal adaptation and voluntary control determine perceptual choices in bistable vision. J. Vis., 8(5), 16.1.

Laing, C. R., \& Chow, C. C. (2002). A spiking neuron model for binocular rivalry. J. Comput. Neurosci., 12(1), 39-53.

Lankheet, M. J. M. (2006). Unraveling adaptation and mutual inhibition in perceptual rivalry. J. Vis., 6(4), 304-310.

Leopold, D. A., Wilke, M., Maier, A., \& Logothetis, N. K. (2002). Stable perception of visually ambiguous patterns. Nat. Neurosci., 5(6), 605-609. 
Levelt, W. J. M. (1965). On binocular rivalry. Institute for Perception RVO-TNO, Soesterberg, The Netherlands.

Moreno-Bote, R., Rinzel, J., \& Rubin, N. (2007). Noise-induced alternations in an attractor network model of perceptual bistability. J. Neurophysiol., 98(3), 1125-1139.

Moreno-Bote, R., Shpiro, A., Rinzel, J., \& Rubin, N. (2010). Alternation rate in perceptual bistability is maximal at and symmetric around equi-dominance. J. Vis., 10(11), 1.1.

Mueller, T., \& Blake, R. (1989). A fresh look at the temporal dynamics of binocular rivalry. Biol. Cybern., $61,223-232$.

Noest, A. J., van Ee, R., Nijs, M. M., \& van Wezel, R. J. A. (2007). Percept-choice sequences driven by interrupted ambiguous stimuli: a low-level neural model. J. Vis., 7(8), 10.1.

Orbach, J., Ehrlich, D., \& Heath, H. A. (1963). Reversibility of the Necker cube. I. An examination of the concept of "satiation of orientation." Percept. Mot. Skills, 17, 439-458.

Pearson, J., \& Brascamp, J. (2008). Sensory memory for ambiguous vision. Trends Cogn. Sci., 12(9), 334-341.

Seely, J., \& Chow, C. C. (2011). Role of mutual inhibition in binocular rivalry. J. Neurophysiol., 106(5), 2136-2150.

Shpiro, A., Curtu, R., Rinzel, J., \& Rubin, N. (2007). Dynamical characteristics common to neuronal competition models. J. Neurophysiol., 97(1), 462-473.

Suzuki, S., \& Grabowecky, M. (2002). Evidence for perceptual "trapping" and adaptation in multistable binocular rivalry. Neuron, 36(1), 143-157.

Taylor, A. L., Cottrell, G. W., \& Kristan, W. B. Jr. (2002). Analysis of oscillations in a reciprocally inhibitory network with synaptic depression. Neural Comput., 14(3), 561-581.

van Ee, R. (2011). Percept-switch nucleation in binocular rivalry reveals local adaptation characteristics of early visual processing. J. Vis., 11(2), 13.1 .

Wang, X. J., \& Rinzel, J. (1992). Alternating and synchronous rhythms in reciprocally inhibitory model neurons. Neural Comput., 4(1), 84-97.

Webster, M. A. (2011). Adaptation and visual coding. J. Vis., 11(5), 2.1.

Wilson, H. R. (2003). Computational evidence for a rivalry hierarchy in vision. Proc. Natl. Acad. Sci. USA, $100(24), 14499-14503$.

Wilson, H. R., Blake, R., \& Lee, S. H. (2001). Dynamics of travelling waves in visual perception. Nature, 412(6850), 907-910.

Wolfe, J. M. (1986). Stereopsis and binocular rivalry. Psychol. Rev., 93(3), 269-282. 